\title{
The role of emotional content in the control of eye movements
}

By

Joseph Phillips

A thesis submitted to Victoria University of Wellington

in fulfilment of the requirements for the degree of

Masters of Science

In Cognitive Behavioural Neuroscience

Victoria University of Wellington

2009 
The anti-saccade paradigm has been a favourite among researchers of attention and the control of eye movements. Most pro/anti-saccade studies have utilized meaningless stimuli, though stimulus meaning is known to have an impact on looking behaviour in free viewing conditions. Here, we explore the role of content in the control of pro/antisaccades by contrasting two alternative views on the impact of emotional stimuli. One view supports an "informativeness" hypothesis, where visual processing is directed towards threatening stimuli, suggesting that RT should be particularly large for negative, high arousal pictures in an antisaccade task. An alternative view emphasizes approach and withdrawal behaviours. Here negative images are thought to encourage avoidance behaviours, causing faster RTs for antisaccades; whereas positive pictures encourage approach behaviours, causing faster RTs for prosaccades. Participants performed an antisaccade task in which they were presented with an image to the left or right visual field and instructed to look at or away from the image. The experimental design included five groups of images, with a factorial combination of valence (positive or negative) and arousal (high or low), and a neutral condition. In Experiments one and two the instruction was given 200 ms before the picture was presented and did not produce any effects of emotional content. Thus, if participants are given advanced notice of the upcoming saccade, the initiation of that saccade is not influenced by the emotional content of the target image. In experiments three and four, the cue was presented $200 \mathrm{~ms}$ after the onset of the target image. This change of SOA provided an effect of emotional content was observed in experiments three and four which was illustrated by slowed RTs for both pro- and anti-saccades. However erotic images appeared to slow down latencies across both saccades which were accompanied by high error rates. 


\section{The role of emotional content in the control of eye movements}

As we move through our environment we are bombarded with an enormous amount of visual information. From this large amount of information hitting our retinas, only a small portion is relevant to us at any particular time. For example when a person is walking along a footpath the images that fall upon the retina may consist of cars driving by, rubbish on the footpath, birds in the sky, or other pedestrians. As they make their way down the footpath they may only focus on relevant stimuli; paper on the footpath to approach (if it is a $\$ 10$ note) or to avoid it (if it is a discarded wrapper), or other pedestrians for possible interaction. These looking behaviours may change depending on a number of factors. One of these factors could be the properties of the stimulus itself. The wrapper on the ground could look like a ten dollar note, or a one hundred dollar note. One would expect the latter to encourage us to inspect it more closely. The pedestrian approaching may be an attractive person of the opposite sex; this again may cause us to stare more intently. Another factor that would affect the looking behaviours could be our current goal. For example if we would like to cross the road, then we would ignore the rubbish and other pedestrians and focus on the cars approaching. On the other hand we may be collecting donations from pedestrians, making them the main focus.

The human eye is designed with one small, central area capable of processing fine detail. To ensure important parts of the scene fall on to this area, allowing that image to be processed in high detail, the eyeball must rotate to position the retina so light from important stimuli falls on this area. These movements are referred to as saccades — fast ballistic movements made about three times a second (Munoz \& Everling, 2004) while we interact with our environment. Using the above example, the individual would be constantly changing the position of their eyes to track oncoming traffic, to judge the height of the curb before they step off or to ensure the crumpled paper on the ground is just a discarded wrapper and not a $\$ 10$ note. This raises the question whether or not the meaning of 
objects and our emotional evaluation of them influence our eye movements. Will we look at a $\$ 10$ note faster than at a discarded wrapper?

\section{The Eye}

The human eye is designed with a central area called the fovea which contains a dense concentration of cone receptors designed to perceive colour. Signals from these cones are sent along the parvocellular pathway which consists of many cells with a small cell body, allowing for little signal loss and high acuity. As we move away from the centre of the retina, rods are increasingly more predominant than cones. Rods are achromatic and their signals summate to allow detection of faint light at the expense of acuity. These averaged signals are then sent along the magnocellular pathway which consists of cells with a much larger cell body to allow for signals to be received from a larger area of visual space. This means that stimuli that fall on the parafoveal area will appear blurry and with poor distinction of colour. The area of light that falls on to the fovea covers about two visual degrees of our total visual field. This means that while the centre of our visual field is perceived with high definition, the remaining visual field is perceived in poor quality. Therefore to direct light from the area of interest to the fovea, the eyes make quick (roughly 20 ms; Carpenter, 1999) ballistic movements called saccades. While viewing a static scene, a person will make about three eye movements a second (Henderson, 2003) that jump from area to area. When a saccade finishes the eye then processes the light that falls onto the retina; this period is called a fixation. To process a scene in detail, fixations need to last at least $150 \mathrm{~ms}$; however to get the gist of a scene viewers only require $40-100 \mathrm{~ms}$ (Rayner, et al., 2009). At a low processing level saccades are made towards contain salient objects, contrasting lines, or large changes in luminance (Land, 1999). However several studies have demonstrated that higher-level processes can affect saccades at early stages.

To investigate individuals' looking behaviours, experimenters have used the free viewing paradigm (for an example see Henderson, 2003). The free viewing paradigm consists of presenting 
the participant with a scene which is typically static, and measuring their saccades and fixations. From these studies it has been found that semantics influence the participants' saccades and their fixations. Yarbus (1967) presented participants with the same scene but asked different questions about that scene. Yarbus found that participants would perform saccades to the objects that were relevant to the question more frequently than to other objects. This finding was extended by Torralba and colleagues (2006) who presented participants with different scenes and asked them to find various objects within that scene. It was found that participants used the global context of the scene to direct their saccades to where the object would most likely be found within that scene; for example when presented with a street scene and asked to find a pedestrian, participants would make saccades around the footpath area, as this is where pedestrians would most likely be found. If asked to find a window however, saccades would be made higher up around the side of a building. This effect has also been shown to not only involve spatial semantics, but also congruent semantics using inconsistent objects - for example a microscope presented in a kitchen scene as opposed to a glass (Henderson, Weeks, \& Hollingworth, 1999). Free viewing studies have demonstrated that not only object semantics affect saccade movement, but the context of the entire scene can also influence where an individual will direct their gaze. However because participants are presented with a large scene both centrally and peripherally, the question is raised as to whether their looking behaviour is influenced by top-down processes driven by semantic information, or bottom-up processes driven by stimuli presented in the peripheral areas. One way to solve this problem is to only present the stimuli to the peripheral area which would allow the investigation of semantic influence from peripheral stimuli.

\section{The antisaccade paradigm}

The antisaccade paradigm (Hallett, 1978) focuses on participants' eye movements when stimuli are presented in the peripheral area. This paradigm compares prosaccades (saccades made towards the target) and antisaccades (saccades made away from the target). These two behaviours 
both appear to be simple saccades but in opposite directions. Yet the mechanisms behind each movement are quite different. Munoz and Everling (2004) introduced a neural circuit pathway which identifies the path of pro- and antisaccades based on a number of lesion, behavioural and animal studies. They found that the production of a saccade begins with the "retino-geniculocortical" pathway. This consists of the retina, lateral geniculate nucleus (LGN) and visual cortex. The retina is responsible for converting light from the environment into electrical signals that is then sent onto the LGN and relayed to the visual cortex. The visual cortex then interprets the electrical signals from images which pass through temporal areas for object recognition and spatial location, are then processed in the frontal brain regions. Other major areas in Munoz and Everling's model are the lateral intraparietal area (LIP), frontal cortex (frontal eye field, FEF; secondary eye field, SEF; and dorsal lateral prefrontal cortex, DLPFC), basal ganglia (caudate nucleus, CN; globus pallidus, GPe; subthalamic nucleus, STN; and substantia nigra pars reticulata, SNpr), superior colliculus (SC), thalamus, cerebellum and spinal reticular formation. Pre-motor activation of a saccade is sent from the reticular formation (an oculomotor portion area in the pons; Segraves, 1992). The reticular formation receives excitatory signals from both the frontal cortex and the SC. The difference between these two pathways is important as the retino-geniculo-cortical pathway to the SC is responsible for reflexive saccades, while the pathway from the frontal cortex to the $\mathrm{SC}$ is controlled by top-down processes responsible for voluntary saccades. The SC receives excitatory signals directly from the lateral geniculate nucleus as well as from visual cortex or LIP. These connections allow quick eye movements to an area of interest due to the bypass of slower higher order processing. While this would be useful in the detection of threatening stimuli, the lack of cortical control means that the individual also has minimal volition over this pathway. The frontal cortex plays a large role in voluntary saccades, particularly via the FEF and SEF areas (Curtis \& D'Esposito, 2003). The SEF is responsible for the sequencing of saccades (Martinez-Trujillo, Wang, \& Crawford, 2003), while the FEF is important in voluntary eye movements (Hanes, Patterson, \& Schall, 1998). The DLPFC is also important for voluntary saccades as it is responsible for spatial working memory 
and executive function. Munoz and Everling's model demonstrates two important features of the saccade process; the first is that the SC and frontal cortex work in parallel. The second is the competitive nature of activation or suppression of the reflexive saccade. These two features are also apparent in another model described by Findlay and Walker (1999).

Findlay and Walker (1999) proposed a framework to describe saccade generation emphasising parallel processing. This model consists of a "where" pathway and a "when" pathway. The when pathway is internally driven and describes the voluntary saccade process, while the where pathway is stimulus driven and represents reflexive saccades. An important concept of the Findlay and Walker Model is the competitive nature of fixation and movement. Movement is described as a spatial map with nodes in each area that activate when stimuli are present in their corresponding areas. When a stimulus is presented and is salient enough to activate a node above a set threshold, then a motor command will be given to move the eye to that particular area. The threshold level is set by the fixation centre. Findlay and Walker propose that the fixation centre is the other side of the movement balance. This centre receives activation from nodes that are sensitive to salient objects within the current fixation area. The fixation area is also influenced by expectancy of peripheral stimuli; the more the stimulus is expected, the higher the activation in the fixation centre, lowering the chances of an eye movement. The top level of the model contains higher level processes. These areas feed down into the remaining levels allowing saccades to be overridden or initiated voluntarily. The Munoz and Everling (2004) model presents the areas involved in the production of a saccade while the Findlay and Walker (1999) model describes how internal and external cues interact to produce a saccade. Both models suggest a threshold that must be met for the ballistic saccade to be made. Activation towards this threshold is also influenced by an opposing force in the form of the inhibiting basal ganglia system in Munoz and Everling model, and the fixation centre in the Findlay and Walker model. The antisaccade paradigm provides a means to examine the competitive nature between activation and suppression of the saccade. 
While the two saccades differ in underlying processes, they also differ in latency and accuracy; antisaccades take longer to initiate and are more prone to errors than prosaccades. This difference can be explained with Munoz and Everling's model. According to the Munoz and Everling (2004) model, when the target is initially presented then the lateral geniculate nucleus and visual cortex will activate the SC, which, with enough activation, will activate a pre-motor signal in the reticular formation, which will result in a prosaccade toward the target. The visual cortex will also send a signal to the DLPFC, FEF and SEF via the parietal cortex. To make an antisaccade, the individual needs to know that they are going to make the antisaccade before the prosaccade is initiated. This will cause an inhibitory signal from the frontal cortex via the basal ganglia to the SC. If this signal is large enough, it will block the excitatory signal from the lateral geniculate nucleus, visual cortex and LIP therefore stopping the initiation of a reflexive saccade towards the target. Once the prosaccade has been stopped, a new saccade is then initiated from the frontal cortex to a newly created landing zone on the opposite side of the visual field from the actual target. This antisaccade is represented in the Findlay and Walker (1999) model by higher level processes increasing activation in the fixation centre which in turn increases the threshold required by the movement centre. According to the two models, making an antisaccade involves many more systems than the prosaccade. This in turn increases latency through extended processing times and increases error rates due to a combination of the increased difficulty for antisaccades and the competitive nature between activation and suppression of reflexive saccades. In summary, antisaccades are more complex and cognitively demanding to perform than reflexive saccades or prosaccades.

The classical antisaccade task presents participants with a central fixation point which is then removed and a target is presented in the left or right visual field. The participant is then required to either look at the target (making a reflexive prosaccade) or to look away from the target (making an antisaccade). The time it takes for the participant to make their first saccade is typically the main dependent variable while the number of erroneous saccades is also measured. This paradigm offers more experimental control than the free viewing paradigm as the antisaccade 
paradigm generates only one saccade from a fixation point to either the target or the opposite side eliminating saccades towards irrelevant areas of the scene. According to the Findley and Walker (1999) model, stimuli in the foveal area affect the chance of a saccade to peripheral areas. In free viewing tasks, a picture is presented that covers the entire visual field. This means that a saccade away from the initial fixation point depends on the content of that fixation area, which will vary depending on the picture and where the participant looks first. Targets in the antisaccade task are only presented to the peripheral areas, allowing a consistent fixation point which will have little effect on saccades away from this area.

While the antisaccade paradigm offers a controlled environment in which to measure saccades, the mechanisms behind prosaccades and antisaccades also have qualities that are useful when studying fundamental cognitive actions. Because antisaccades require a large amount of inhibition to correctly perform, accuracy and latency of antisaccade has become popular measures of cognitive inhibition used in a number of different areas: schizophrenia (Reuter \& Kathmanm, 2004; Martin, et al., 2007; Campanella \& Guerit, 2009), Parkinson's Disease (Chan, et al., 2005) substance abuse (McNamee, et al., 2008) and personality (Nguyen, Mattingley, \& Abel, 2008). The current study will use this inhibition to investigate how pro- and antisaccades are affected by the content of the stimuli.

\section{Emotional stimuli}

The antisaccade task traditionally employs a meaningless symbol as a target; however the current study will be interested in how the content of the target affects task performance. To achieve this, targets will consist of emotionally charged pictures selected from the International Affective Picture System (IAPS; Lang, Bradley, \& Cuthbert, 1997). These images vary in valence and arousal. Emotional pictures will be used for two reasons; the first is that interpretations of emotional pictures are generally consistent across individuals and cultures (Verschuere, Crombez, \& Koster, 2001; Yuxia, \& Yuejia, 2004; Ribeiro, Pompeia, \& Bueno, 2005); the second reason is 
because emotional information is typically prioritised over other incoming information (Müller, Anderson, \& Keil, 2008). Emotional pictures have been effective when inserted into a number of different visual paradigms in a range of studies looking at attention, affective disorders, phobias and neglect.

\section{Allocation of Attention}

Green and colleagues (2003) studied threatening pictures using a non-clinical population in a free viewing paradigm. The emotional pictures participants were presented with consisted of either threatening or non-threatening images. The researchers noted that for threatening pictures participants employed different scanning techniques than for non-threatening pictures. The results illustrated longer scanning paths with more fixations of longer duration when participants were presented with threatening pictures. Green and colleagues suggested this was due to "vigilance" where by participants employed these strategies to ensure a high amount of visual information was obtained from the potential threat. Pflugshaupt et al.'s (2007) study used a free viewing paradigm in which participants were presented with two pictures simultaneously in the left and right visual fields. One picture was of a spider, while the other picture was a non-fearful image (e.g. a butterfly). Participants were then allowed to look at either picture for as long as they liked while the number and area of their fixations were recorded. Pflugshaupt and colleagues found similar behaviours to Green and colleagues with their non-clinical participants. However this seemed to be modulated by the coping strategies of the individuals; participants with avoidant strategies would look towards the non-fearful image more often than non-avoidant participants. They also found that the phobic participants' eye movements were much faster overall than the non-clinical participants; however reaction time for the first saccade was not mentioned in the study. Faster reaction times of the first saccade either towards or away from the fearful image would have indicated whether phobic participants can detect the fearful stimuli in their peripheral vision and avoid or approach it before they are consciously aware of its presence. 
Emotional pictures have also been used in visual search tasks to investigate how anxious or phobic patients process threatening pictures (Flykt \& Caldara, 2006; Horley, Williams, Gonsalves, \& Gordon, 2003; 2004; Öhman, Flykt, \& Esteves, 2001). Here participants are presented with an array of items and are instructed to find an "oddball" item. The stimuli used in these studies differ emotionally — finding a fearful face in a group of happy faces or vice versa; or a fear invoking stimulus (e.g. spider) in a group of non-fearful stimuli (e.g. flowers). Time taken to find the item of interest is typically the dependent variable in these studies. Shorter reaction times demonstrate a greater pull of attention to the item of interest compared to other items in the array. These studies typically find that fearful stimuli are found much faster than neutral stimuli, supporting the idea that fearful pictures attract attention, as saccades require attention to their target area (Godijn \& Theeuwes, 2003). Thus participants look at fearful pictures faster than neutral pictures. This effect is also found in more controlled paradigms such as the dot-probe task.

Macleod, Mathews and Tata (1986) took Posner and colleagues' (1980) cueing task and modified it to create the dot-probe task. Instead of presenting one cue to one visual field as in the Posner et al. task, Macleod and colleagues presented two words simultaneously in each visual field. This was then followed by a target which appeared where one of the pictures was presented. The idea behind this procedure was to investigate how different words attract exogenous attention, an attention mechanism that is pulled towards abrupt peripheral stimuli, with little control from the individual. Nummenmaa, Höynä and Calvo (2006) used this methodology but replaced words with emotional pictures as cues. It was found that participants were faster to identify the target when it was presented where an emotional picture was initially presented and slower when the target was presented to the opposite side. This suggests that attention is not just attracted to threatening pictures as shown in the visual search tasks, but it is attracted to pictures with emotional content. This bias toward emotional stimuli was also found in unilateral neglect patients (Vuilleumier \& Schwartz, 2001) and anxious patients (Fox, 2002; Broomfield \& Turpin, 2005). While such data suggest that attention is attracted to emotional pictures, the question remains whether emotional 
pictures actually affect orienting of attention or whether participants simply choose to focus on the emotional picture as it is more interesting than the neutral picture.

Schupp and colleagues (2004) investigated participants' event related potential (ERP) responses to emotional pictures. Participants in this study were presented with pictures that varied in emotional content. These pictures fell into three categories: pleasant (erotic scenes, generic sports scenes), neutral (household items) and unpleasant (threatening pictures, pictures of pollution and disease, pictures of injuries). Participants had their ERP monitored while they were observing each picture. The study found that emotional pictures produced stronger late positive potentials than neutral pictures. This suggests that the emotional pictures invoked stronger attention processes than neutral pictures. This study also found that emotional pictures that depicted sex or violence (high arousal pictures) also invoked higher startle reflexes than other emotional pictures. These results support the idea that not only are emotionally charged images are processed differently to neutral pictures, but the responses to the emotional images are relative to their intensity.. The above studies have shown that emotional pictures have a robust effect on attention, where both negative and positive emotional pictures tend to attract attention towards them compared to neutral pictures. However there are a number of studies that suggest individuals look at emotional pictures using approach and withdrawal behaviours.

\section{Approach and Withdrawal}

Studies looking at approach and withdrawal behaviours using emotional pictures and visual paradigms typically find that positive pictures are looked at faster than neutral pictures which are faster than negative pictures. Negative pictures are looked away from faster than neutral pictures which are faster than positive pictures. When comparing pro- and antisaccade to approach and withdrawal, making a prosaccade would be considered an approach behaviour as this will increase incoming information; while making an antisaccade would be considered a withdrawal behaviour as it stops incoming visual information regarding the stimuli. Gray (1990) describes two systems that 
address these two opposing types of behaviour. The first system is the behavioural approach system (BAS) and is responsible for encouraging an organism to approach a possible rewarding stimulus (e.g. food, shelter or a mate). The BAS system is sensitive to signals of upcoming reward and the absence of a punishment. The opposing system is the behavioural inhibition system (BIS). This system is sensitive to possible threats or punishments (e.g. predators, illness, and a dominant con-specific) and encourages the organism to avoid the particular stimuli. Input for this system according to Gray includes signals of punishment or non-reward, novel stimuli and stimuli that accompany innate fear. The anatomy for the BAS involves a number of systems which are also involved in learning. As BAS is always aimed towards a goal, and working memory is an important component of goal-seeking behaviour, the DLPFC plays a large part in the BAS (Davidson, 1998). The nucleus accumbens is also involved to provide reinforcement of the goal. The final area mentioned by Davidson is the medial prefrontal cortex, as this area is responsible for maintaining behavioural-reinforcement contingencies. The BIS relies on areas related to fear and disgust. These include the amygdala, which is important for processing fearful stimuli (LeDoux, 2000) and also may be involved in associating the stimuli with negative consequences. The BIS system also includes the basal ganglia and hypothalamus, which are responsible for autonomic reflexes (Smith \& DeVito, 1984). Do these two systems differentially affect the looking behaviour when observing emotional pictures?

Stormark and Hugdahl (1996) begin to answer this question by employing Posner's spatial cueing task with classically conditioned stimuli. Participants were initially presented with two emotionally meaningless cues. During the second phase of the experiment one of the cues was paired with an aversive noise (90db white noise) presented through headphones. This created a conditioned stimulus. Participants then ran through the spatial cueing task again with the same stimuli. The study found increased skin conductance responses for the conditioned stimuli, but more importantly, while reaction times were faster for valid than invalid trials for the nonconditioned stimuli, this effect was not found for the conditioned stimuli. What this suggests is that the attraction of attention that was present for the non-conditioned stimuli was removed for the 
conditioned stimuli. The authors suggest that this maybe due to attention avoidance, thus removing the advantage typically gained from the valid trials. A similar effect was found by Mackintosh and Mathews (2003). However in this study the cues were paired with positive and negative emotions. Two groups of participants were presented with ambiguous line drawings. The first group was presented with pictures paired with a positive meaning; while the second group received the same pictures paired with a negative meaning. This provided identical images with opposite valence. The participants were then given a dot-probe task where a positively conditioned picture was presented with a negatively conditioned picture, followed by a target presented to the left or right of the screen. The results indicated longer RTs when the target appeared where the negative picture was, suggesting that the participants' attention was on the positive picture, supporting avoidance of negative stimuli, and approaching positive stimuli. The Stormark and Hugdahl (1996) and Mackintosh and Mathews (2003) studies both demonstrate that participants avoid moving their attention towards stimuli that have either been paired with an aversive stimuli or paired with a negative meaning. These findings contradict findings in the studies already covered that support a strong pull of attention toward emotional pictures, which is especially strong for negative emotions such as threatening images.

\section{Emotional Pictures and Antisaccades}

This thesis so far has introduced saccades and the basic mechanisms behind them and the free viewing paradigm which has been used to study them. However visual attention plays a large role in the planning and execution of saccades. There are a number of paradigms that look at the components of visual attention, and how these are affected by emotional stimuli. An alternative theory was also covered with approach and withdrawal behaviours. This theory suggests that we treat visual stimuli in an appetitive or aversive manner, approaching images depicting positive emotions while avoiding images depicting negative emotions. To investigate the affect of emotional content on eye movements, the antisaccade paradigm is presented as an alternative paradigm that 
will offer a more controlled environment to study emotional influences on eye movements. This paradigm has already been applied in this field with interesting outcomes.

Gerdes, Pauli and Alpers (2009) used a paradigm similar to the antisaccade paradigm. Here spider phobic participants were simultaneously presented with a neutral picture and a picture of a spider. The participants were instructed to identify each picture while maintaining central fixation and then to look at either the neutral picture (i.e. antisaccade) or the fearful picture (i.e. prosaccade). They study found that participants took longer to look at the target image when it was a neutral picture and they had to identify the spider picture. While this study suggests an attraction of attention towards the threatening pictures, the participants were only presented with neutral or threatening pictures. This leaves the question as to whether attention is attracted to threatening pictures, or to emotional pictures in general.

Kissler and Keil (2008) examined how emotional pictures affected prosaccades and antisaccades. This study presented participants with two groups of pictures, positive valence and negative valence, in their left or right visual field. Participants were then required to either look at the picture, making a reflexive or prosaccade, or to look away from the picture, making an antisaccade. There were also two stimulus onset asynchrony (SOA) conditions; the first presented the image $200 \mathrm{~ms}$ after a central fixation point was removed; the second condition presented the image simultaneously with the removal of the fixation point. The study found no interaction between saccade type and picture content and no interaction between the SOA conditions and saccade type. There could be a number of reasons behind these results. The results could be due to the lack of control over the images used. The pleasant picture group consisted of high and low arousal images, while the negative group contained only high arousal pictures. There was also an imbalance of picture content across the groups, with all of the positive pictures containing people, the negative group containing both animal and people and the neutral group containing faces and inanimate objects. The results could also be due to the single task blocks, allowing participants to 
become adept to making antisaccades. Finally, the lack of interaction could be due to the SOAs used. As both require the participant to make the saccade before they have a chance to process the picture, the picture content may not be processed to the point of being able to separate the positive pictures from the negative pictures.

The current study will use a design similar to that of the Kissler and Keil (2008) study. However the pictures used will be balanced in valence and arousal to create five groups, and arousal will be included in the analysis. While experiments one and three will use single task blocks, experiments two and four will employ mixed task blocks. For this reason an instructional colour cue will be introduced: green to signal a prosaccade and red to signal an antisaccade. Finally the simultaneous SOA will be replaced with a -200 ms SOA. This means that the image will be presented peripherally 200 ms before the instructional cue; however central fixation will be maintained until the instructional cue is presented. This will allow the participants to process the picture briefly before they are required to make the saccade.

The current study will generate three predictions. The first is that emotional content will have a larger effect on antisaccades than prosaccades. According to the Munoz and Everling (2004) and the Findlay and Walker (1999) models, antisaccades rely heavily on the frontal areas, making these more prone to interference than prosaccades are. The second prediction is that this effect will be larger for high arousal pictures than for low arousal pictures. This prediction is supported by Rozenkrants and Polich (2008) who looked at participants ERP responses to various emotional pictures in an oddball task. They found stronger responses for high arousal pictures than for low arousal picture. This suggests that the more intense high arousal pictures may activate semantic representations faster than the less intense low arousal pictures. The final prediction is responses will be consistent with mechanisms controlling approach and withdrawal. This means that in relation to neutral pictures, positive pictures will have shorter latencies for prosaccades and longer 
latencies for antisaccades. Negative pictures will have longer latencies for prosaccades and shorter latencies for antisaccades compared to neutral pictures.

\section{Experiment One}

Design and participants

Experiment one employed a 2 (saccade) $\times 5$ (emotional content) $\times 2$ (visual field) design. Participants were first-year psychology students at Victoria University of Wellington, New Zealand, who had normal or corrected-to-normal vision, and had consented to being exposed to explicit pictures. There were 24 participants ( 15 female) in each Experiment. The participants' mean age was 19.5 years. Each participant was tested individually, and was awarded credit towards their course requirements.

\section{Apparatus and Stimuli}

Each experiment was presented on a Windows XP computer with a 21" monitor set to a 1280 by 1024 resolution. The experiments were programmed using SR Research Experiment Builder (SR Research). Eye movements were tracked using the Eyelink 1000 Tower Mount Head Supported System (SR Research Ltd., Ontario, Canada), which positioned the head $57 \mathrm{~cm}$ from the screen. The eye-movement data were recorded on a separate computer.

Stimuli were drawn from the International Affective Picture System (IAPS; Lang, Bradley, \& Cuthbert, 1997), which consists of 824 images and has established ratings of valence, arousal and dominance. These ratings have shown to be consistent across several cultures (Verschuere, Crombez, \& Koster, 2001; Yuxia, \& Yuejia, 2004; Ribeiro, Pompeia, \& Bueno, 2005). One hundredand-sixty pictures were selected for the current study (for a full list see table 1). These pictures were chosen to compose five different stimulus sets: positive valence, high arousal (PH; e.g., erotic or extreme sports pictures), positive valence, low arousal (PL; e.g., smiling faces or pictures of food), negative valence, high arousal ( $\mathrm{NH}$; e.g., mutilated people or pictures of threatening situations), 
negative valence, low arousal (NL; e.g., pollution or pictures of unhygienic situations) and a neutral stimulus set (NE; e.g., furniture or abstract pictures). Mean valence and arousal ratings are presented in table 2. Independent sample t-tests were used to confirm that: 1) PH and PL were significantly different in arousal, but not in valence; 2) NH and NL were significantly different in arousal, but not in valence; 3) PH and NH were significantly different in valence, but not in arousal; and 4) PL and NL were significantly different in valence, but not in arousal. The neutral stimulus set was significantly different from all other sets in both valence and arousal. Erotic images were selected to ensure that there were no sex differences in ratings in valence and arousal. Item order was pseudo-random across participants to ensure that no two participants were given the same order of items, and that condition (visual field, valence or arousal) was not repeated more than three times in a row.

The stimulus sets were then divided and rearranged into four lists. Each list consisted of eight items from each stimulus set (four presented to the left visual field, four to the right visual field), giving a total of 40 items for each list. The four lists were created by duplicating the original list and alternating which items were presented in anti- or prosaccade trials, as well as alternating the visual field in which items were presented. The construction of lists was conducted in this way so as to ensure that each item was presented equally often as a target for a pro- or antisaccade in the left or right visual field across all participants. The four lists were randomly assigned to each participant while the list order for each participant was pseudo-random to ensure that no two participants received the same list order. Each participant ran through 320 trials, divided into eight blocks of 40 . Each block contained only prosaccades or antisaccade (single task blocks). The first 160 trials were repeated with their saccade condition switched to make up the final 160 trials.

Each picture was in full colour with a 1024 × $768(30 \times 38.5$ visual degrees $)$ resolution originally, but reduced to $344 \times 258$ (12.8 $\times 9.8$ visual degrees)to ensure the participant would have to make a significant saccade to look at the picture when it is presented to one visual field. The 
overall luminance of each picture was measured. Ten pictures $(2141,2890,9415,2688,4681,1201$, $1525,4689,7009$ and 7390 ) were either brightened or darkened to level out the luminance of the stimulus set using Photoshop (Adobe Systems Incorporation, 2007)

\section{Procedure}

Participants were asked to read and complete a consent form that outlined the nature of the images used in the study and reminded the participant that they could withdraw from the study without penalty. Participants were then invited to sit in a comfortable position in front of the eyetracker, which was then adjusted to maintain comfort while still producing accurate readings. After the eyetracker had been adjusted the participant was asked to go through the instructions at their own pace. After the participant had read the instructions, the experimenter repeated the main points and answered any questions. A practice block of eight trials was also presented to ensure the participant understood the task.

Before each block the participant underwent a calibration task followed by a validation task to ensure accurate readings from the eyetracker. This was repeated until the average error was less than 0.5 visual degrees and the maximum error was less than 1 visual degree. Each trial was presented on a grey background and began with a black fixation point measuring $0.8^{\circ}$ and presented in the centre of the screen. In order to reduce anticipatory effects, the fixation point was presented for $750,1000,1250$ or 1500 ms. Duration was pseudo-randomly assigned to each trial to ensure no value was repeated more than three times in a row. The fixation point was then replaced by the instructional cue (IC), which was presented for $200 \mathrm{~ms}$. The IC had the same dimensions as the fixation point, but was either red or green. A green IC indicated that the trial required a prosaccade while a red IC indicated an antisaccade. This was followed by the target image (see diagram 1A).

The target image screen contained the IC, the target image, and an antisaccade interest area, which was invisible to the participant. The target image measured $13.68^{\circ}$ by $10.26^{\circ}$ and was 
positioned $3.94^{\circ}$ from the edge of the fixation point. The antisaccade interest area measured $21.87^{\circ}$ by $33.80^{\circ}$ and was positioned $1.75^{\circ}$ from the fixation point. The participant was then required to either look at the picture (if the IC was green; prosaccade) or look to the opposite side of the screen (if the IC was red; antisaccade). The trial was complete when the participant looked at the picture or if their gaze entered the antisaccade interest area. After each block the participant was encouraged to have several long blinks, and at the midpoint of the total experiment, the participant was given the opportunity to have a break.

Results

Saccades with a reaction time of less than $50 \mathrm{~ms}$ were discarded as anticipatory responses. Saccades that had horizontal amplitude of less than 2 visual degrees were also discarded as saccades smaller than this did not leave the fixation area, and the purpose for their initiation is ambiguous. Finally saccades that had smaller velocity than 35 visual degrees per second were also discarded as this is the minimum speed used in previous studies to distinguish actual saccades from drifts. The first saccade of each trial that met the above requirements, regardless of whether they were correct or not were used in the main dataset; however only correct saccades were used in the following analysis.

\section{Reaction times}

The means for reaction times of each condition for experiment one are presented in table 3 , mean errors are presented in table 4. A 5(emotion) $\times 2$ (saccade) $\times 2$ (visual field) repeated measures ANOVA was run on data from experiment 1. Prosaccades $(M=162.48 \mathrm{~ms}, \mathrm{SD}=46.48)$ were made significantly faster than antisaccades $(M=229.00 \mathrm{~ms}, \mathrm{SD}=54.82), F(1,23)=77.36, p<.001, \mathrm{n}_{\mathrm{p}}{ }^{2}=$ .77 (figure 1). However there was no effect of emotional picture on saccade $F(4,92)=0.38, p=.82$, $\eta_{p}^{2}=.016$. No other effects were observed.

\section{Errors}


Another repeated measures ANOVA was run on the errors made on each condition. While there were more errors made on the antisaccade trials $(M=13.38 \%, S D=10.88 \%)$ these were not significantly higher than the prosaccade trials $(\mathrm{M}=6.72 \%, \mathrm{SD}=7.06 \%), F(1,23)=24, p<.001, \eta_{\mathrm{p}}{ }^{2}=$ .51.

\section{Discussion}

The results from experiment one support the direction effect found in Hallett's (1978) study in that prosaccades were made much faster than antisaccades. Analysis of errors also supports Hallett's study as there were significantly more errors in the antisaccade task than the prosaccade task. While there was an effect of saccade, there were no significant differences between the emotion images. One reason for this could be the task difficulty. As each block consists of only prosaccades or only antisaccades; participants may become quite adept to performing each task. To counter this effect experiment two will use mixed task blocks.

\section{Experiment Two}

Experiment one contained blocks of either prosaccades or antisaccades (single task blocks). As this design failed to show effects of emotion type, the blocks in experiment two will contain both prosaccades and antisaccades (mixed task blocks). Literature suggests that the mixed task block will create a larger load on working memory, decreasing attentional resources (Hester \& Garavan, 2005). This will increase the difficulty of the task making antisaccades harder to perform (Godijn \& Kramer, 2007) increasing latencies and errors. This may also make them more prone to effects of emotion type that were not detected in the first experiment.

\section{Design and participants}

Experiment two used a similar design to experiment one, 2 (saccade) $\times 5$ (emotional content) x 2 (visual field). Participants were drawn from the same pool as experiment one, however participants were only selected on the proviso that they did not take part in experiment one. The 
mean age of the population used in experiment two was 18.83 years and consisted of 13 females, 11 males. Participants were given credit towards their course completion for participating.

\section{Apparatus and Stimuli}

Experiment two was designed and run using the same programs and setup as experiment one. Stimuli used were the same pictures used in experiment one. Lists were constructed in a similar fashion to experiment one; however experiment two included the saccade condition within each list. This condition was counterbalanced across the remaining conditions and trial order was pseudo-randomise to ensure that no condition was repeated more than three times in a row.

\section{Procedure}

Participants were tested in the same room using the same computer and eyetracker as experiment one. The procedure was identical to experiment one (diagram $1 \mathrm{~A}$ ) except that trials within blocks alternated between prosaccades and antisaccades in a random fashion.

\section{Results}

Data was reduced using the same parameters as experiment 1 . Mean reaction times for experiment 2 are presented in table 3, mean error rates are presented in table 4.

\section{Reaction Times}

A repeated measures ANOVA on reaction time data produced a significant difference between prosaccades and antisaccades, with prosaccades $(\mathrm{M}=219.85 \mathrm{~ms}, \mathrm{SD}=71.85)$ initiated significantly faster than antisaccades $(M=273.89 \mathrm{~ms}, S D=79.17), F(1,23)=59.41, p<.001, \eta_{p}{ }^{2}=$ .71 (figure 3). However, there were no effects of emotional picture type, and no interaction with saccade type.

Errors 
Analysis of errors yielded a significant difference across the two saccades, with prosaccades $(M=6.72 \%, S D=7.06 \%)$ having a lower error rate than antisaccades $(M=13.38 \%, S D=10.88 \%), F(1$, $23)=39.87, p<.001, \eta_{p}^{2}=.63$ (figure 4).

\section{Discussion}

Data from experiment two also demonstrates the direction effect found in experiment one. More importantly, reaction times for both pro- and antisaccades are larger in experiment two than experiment one. This indicates that the mixed task blocks were indeed more difficult than the single task blocks. The manipulation has also produced a significant difference between error rates for each saccade with antisaccades having twice the error rate of prosaccades. However there was still no effect of emotional content for each saccade type. One possible reason for this could be the order of the IC and the image presentation. Thus far the IC has been presented 200 ms before the image. This means that during the antisaccade trials participants have $200 \mathrm{~ms}$ to inhibit the SC before the picture has been presented; giving a large advantage to antisaccades. This also may have resulted in participants using the presentation of the image as a temporal cue to trigger the onset of the ballistic eye movement. Experiments three and four are designed to establish an effect of emotional content by deviating away from the traditional antisaccade paradigm.

\section{Experiment Three}

To determine whether emotional images can influence the generation of saccades when they are viewed peripherally; experiment three will introduce a -200 ms SOA. Images will be presented $200 \mathrm{~ms}$ before the IC, allowing the image to be briefly processed before the eye movement can be programmed.

\section{Design and Participants}

The design of experiment three followed experiment one, 2 (saccade) x 5 (emotional content) $\times 2$ (visual field). Participants were drawn from the same pool as experiment one and two; 
however participants were only selected on the proviso that they did not take part in either experiment one or two. The mean age of the population used in experiment three was 20.83 years and consisted of 17 females, 7 males. Participants were given credit towards their course completion for participating.

\section{Apparatus and Stimuli}

Experiment three used the same apparatus as experiment one and two. The stimuli used were also the same pictures used in experiment one and two. Lists used were identical to experiment one, as both experiments manipulated saccade type between blocks.

\section{Procedure}

The procedure for experiment three was similar to experiment one. In experiment three, however, instead of the instructional cue being presented 200 ms before the image, the image was presented $200 \mathrm{~ms}$ before the instructional cue (diagram 1B). Participants were instructed to maintain fixation on the initial fixation point until it changed into the instructional cue. If this fixation was broken before the instruction cue was presented then an error message was presented and the trial was restarted. Trials that were repeated were excluded from analysis.

Results

Data was reduced using the same parameters as experiment 1 and 2 . Mean reaction times are presented in table 3 and mean errors in table 4.

\section{Reaction Times}

A repeated measures ANOVA on reaction times revealed a significant difference between saccade types, however this was inverted from the previous two experiments with antisaccades $(\mathrm{M}=$ $266.02 \mathrm{~ms}, \mathrm{SD}=83.40)$ performed faster than prosaccades $(\mathrm{M}=300.14 \mathrm{~ms}, \mathrm{SD}=89.16) F(1,23)=$ $15.20, p<.01, \eta_{p}{ }^{2}=.40$. While there was no significant difference across the emotional picture types 
there was a two way interaction of emotion type and saccade $F(4,92)=3.39, p<.05, \eta_{p}{ }^{2}=.13$ (figure 5). To investigate this interaction in more detail, post hoc analysis was performed.

Additional analysis examined the facilitating or interfering effects each emotional picture group had on either the pro- or antisaccade. Reaction times from each of the four emotional image groups were subtracted from reaction times from the neutral emotional image group to provide an index of facilitation (positive values) or interference (negative values). The data suggest that the positive images interfered with the initiation of both types of saccades, while the negative images facilitated the initiation (table 6). This effect was shown to be significant $F(1,23)=4.68, p=.04, \eta_{p}{ }^{2}=$ .17; however, it further interacted with saccade type. To investigate this interaction the saccades were analysed separately. An effect of valence was observed in the prosaccade condition $F(1,23)=$ $6.43, p=.018, \eta_{p}^{2}=.22$ with positive images interfering with saccade initiation and negative images facilitating saccades (figure 13). To test if the facilitation and interference was significant, One sample t-tests were then run on the positive valence images $t(23)=-2.10, p=.047$ and the negative valence images $t(23)=0.90, p=.38$. Neither test maintained significance after a Bonferroni correction (alpha $=.025)$. Similar analysis on the antisaccade condition found no significant effects of valence or arousal (figure 14).

The results from the above tests suggest that the effect is mainly driven by the positive valence, high arousal group. This prompted a closer look into this picture set. Pictures from this image group can be sorted into two main categories: erotic images and non-erotic images. Using an one-way ANOVA it was found that the erotic images $(M=313.82 \mathrm{~ms}, S D=22.27)$ had longer reaction times for the prosaccade condition than the non-erotic pictures $(M=279.95 \mathrm{~ms}, \mathrm{SD}=17.07), F(1,30)$ $=9.22, p<.01, d=1.4$. The negative valence, high arousal group could also be dissected in a similar fashion, creating a set of pictures that elicit fear, and a group that elicit disgust. While there were no significant differences in reaction times between these two sets this could be attributed to the imbalanced number of pictures in each group with fear containing 26 pictures; and the disgust group 
containing only 6 pictures. As erotic pictures are the driving force behind the interaction between saccade and emotion type, further analysis was run to compare data from the erotic group, fear group and the neutral group. Means and errors of these groups are displayed in table 5. A repeated measures ANOVA gave a significant effect of condition $F(2,46)=7.86, p=.001, \eta_{p}{ }^{2}=.26$, with erotic pictures having longer reaction times than the other two groups for the prosaccade condition, but not for the antisaccade condition (figure 7).

\section{Errors}

A repeated measures ANOVA was run on error rates, no significant differences were found between the saccade tasks or across the conditions (figure 6). Separating the emotion groups into erotic, fearful and neutral; a repeated measures ANOVA on the error rates exhibited an effect of emotion type with the erotic group showing the most amounts of errors followed by the fear group, $F(2,46)=804.67, p<.001, \eta_{p}{ }^{2}=.97$ (figure 8). There was also an interaction of emotion type and saccade $F(2,46)=8.43, p=.001, \eta_{p}^{2}=.27$

\section{Discussion}

Data from experiment three illustrate longer latencies for both pro- and antisaccades than found in experiment one and two. This indicates another increase in task difficulty. However the data also indicates longer latencies for prosaccades than for antisaccades. This contradicts not only results from experiments one and two; but also previous studies using the antisaccade paradigm (Hallett, 1978; Ansari et al., 2008; Grodjin et al., 2007). One possible reason for this could be the inhibition of return (IOR) phenomena. In which individuals are slower to return to an area that was previously attended (Klein, 2000). During the prosaccade trials the participants' exogenous attention will be briefly drawn towards the image, ultimately leading to inhibition at that location. For the saccade to the image to be made, attention must return to the inhibited image, leading to increased reaction times. For the antisaccade task this is not the case as attention must be pushed 
towards a new location before the saccade in opposite direction can be made, not only avoiding IOR, but also reducing latencies for the antisaccade task.

There was a significant interaction of emotional content on saccade type. This interaction suggests that participants took longer to look at positive pictures than negative pictures. Participants were also quicker to look away from positive pictures than negative pictures. Further analysis on interference and facilitation of each emotional image group supported this with positive images interfering with prosaccades and negative images facilitating them. However, none of the emotional groups produced enough interference or facilitation on the initiation of the prosaccade to maintain significance after a Bonferroni correction.

\section{Experiment Four}

To increase task difficulty experiment four employed mixed task blocks with the $-200 \mathrm{~ms}$ SOA.

\section{Design and Participants}

The design of experiment four followed the three previous experiments, 2 (saccade) $\times 5$ (emotional content) $\times 2$ (visual field). Participants were drawn from the same pool as the previous experiments; however participants were only selected on the proviso that they did not take part in either experiment one, two or three. The mean age of the population used in experiment three was 18.33 years and consisted of 13 females, 11 males. Participants were given credit towards their course completion for participating.

\section{Apparatus and Stimuli}


Experiment four used the same apparatus as previous experiments. The stimuli used were also the same pictures used in experiment one, two and three. Lists used were identical to experiment two, as both experiments manipulated saccade type within blocks.

\section{Procedure}

Experiment four used the same procedure as experiment three (diagram 1B), however saccades were alternated within blocks in a pseudo-random order ensuring that no condition was repeated more than three times.

Results

Data for experiment four was reduced using the same parameters as the three previous experiments. Mean reaction times for each condition are presented in table 3; mean errors are presented in table 4.

\section{Reaction Times}

A repeated measures ANOVA was run on reaction times which found that prosaccades $(M=$ $362.37 \mathrm{~ms}, \mathrm{SD}=78.42)$ were performed significantly faster than antisaccades $(\mathrm{M}=408.34 \mathrm{~ms}, \mathrm{SD}$ $=95.51), F(1,23)=24.43, p<.01$ (figure 9). There was also a significant effect of emotion type $F(4$, $92)=5.22, p<.01$, however as there was no interaction between saccade type and emotion type, reaction times from both saccades were collapsed together to give five emotion type groups. A oneway ANOVA failed to show a significant difference in reaction times between the conditions.

Facilitation and interference effects were analysed using the same method as used in experiment three (table 6). Initial analysis suggests that positive valence images produced more interference across both saccades than negative valence images $F(1,23)=5.15, p=.033$. A similar effect was found with arousal $F(1,23)=18.91, p<.001$, with high arousal pictures creating more interference than low arousal pictures. However, these effects were qualified by a three way 
interaction of saccade type, valence and arousal which approached significance $F(1,23)=3.99, p=$ .06. This prompted further analysis to be performed on each saccade separately. A repeated measures ANOVA examining effects of valence and arousal for the prosaccade condition produced a significant effect of valence $F(1,23)=7.35, p=.012$ with positive images interfering with saccade initiation and negative images facilitating initiation (figure 15). The amount of interference and facilitation was non-significant, $t(23)=1.81, p=.08$ and $t(23)=-0.13, p=.90$ respectively. There was no significant effect of arousal $F(1,23)=1.14, p=.30$. Similar analysis on the antisaccade condition produced an effect of arousal $F(1,23)=10.16, p=.004$, which further interacted with valence $F(1$, $23)=4.32, p=.049$. High arousal images interfered with the inhibition of the reflexive saccade, producing longer RTs for the antisaccade. This was only apparent in positive valence images (figure 16). Further analysis showed significant interference of the high arousal group $t(23)=-2.43, p=.023$ which was maintained after a Bonferroni correction.

The high arousal groups were dissected into the three subgroups as experiment 3 , and the erotic and fearful groups underwent further analysis. Means and errors of these two groups and the neutral group are presented in table 5. A repeated measures ANOVA showed that the erotic group had significantly longer reaction times than the fear and the neutral groups $F(2,46)=15.02, p<.001$ which was consistent across both saccade conditions (figure 11). The two saccade conditions were then collapse across the emotion types and paired t-tests were performed on each pair. The erotic group $(M=414.73 \mathrm{~ms}, \mathrm{SD}=81.06)$ had significantly slower reaction times than the fear group $(\mathrm{M}=$ $379.78 \mathrm{~ms}, \mathrm{SD}=70.32)$ and the neutral group $(\mathrm{M}=379.03 \mathrm{~ms}, \mathrm{SD}=74.86), t(23)=4.05, p<.001$ and $t(23)=4.66, p<.001$ respectively (figure 11 ).

\section{Errors}

A repeated measures ANOVA on error rates gave an effect of saccade type, with antisaccades $(M=15.73 \%, S D=14.12 \%)$ producing more errors than prosaccades $(M=10.94 \%, S D=$ 12.53\%) $F(1,23)=5.19, p<.05$; but no differences across emotion types (figure 10). A repeated 
measures ANOVA was run on the percentage of errors in each separated condition and found an effect of emotion type, $F(2,46)=544.89, p<.001$; but no interaction between saccade and emotion type. When the two saccade conditions are collapsed into each other the erotic group had significantly more errors than the fear group, $t(23)=17.10, p<.001$; and the neutral group, $t(23)=$ $32.60, p<.001$, while the fear group had significantly more errors than the neutral group $t(23)=$ $16.04, p<.001$ (figure 12).

\section{Discussion}

Overall latencies in experiment four were longer then the three previous experiments, indicating an increase in difficulty. Prosaccade trials also had significantly shorter latencies than antisaccades, which while supporting experiments one, two and previous studies, contradicts experiment three which used the same SOA. This difference in the direction of the effect may be due to the single vs. mixed task block design. In experiment three participants knew what each task was after the first trial in the block allowing them to relax and let their attention drift from fixation. In experiment four participants' were unable to predict the task of their current trial. This encouraged participants to be aware of where they were attending making them less prone to IOR. An effect of emotional content was also found which was driven by the positive valence, high arousal group. Analyses of facilitation and interference effects show positive valence images interfering with both saccades more than negative images. It was also found that high arousal images interfered more than low arousal. When each saccade was analysed separately the effect appeared to be driven by high arousal, positive valence images, which were the only images that provided significant interference for the antisaccade task. Further analysis of this image set suggested that the erotic pictures in this group were responsible for the difference in RTs. Analysis of error rates also show that the erotic group also had the most errors, followed by the fearful group and the neutral group. 


\section{General Discussion}

Data from experiment one and two show that prosaccades were not only made faster than antisaccades, but the prosaccade task also produced fewer errors. There were however no differences between the emotion groups. Data from experiment three presented a reverse effect to that of experiment one and two where antisaccades were performed faster than prosaccades with difference in error rates between the two tasks. There was also an interaction of emotion group and saccade type present in experiments three and four; driven by the $\mathrm{PH}$ group. Comparing latencies from the neutral images and the emotional images provided indexes of facilitation and interference. Data from experiment three suggests that positive images interfered with, and negative images facilitated prosaccades. Antisaccades were interfered with by negative images. This pattern was not present in experiment four as positive images created interference across both saccades. The PH group was also the only group that caused significant interference. When the emotion groups were broken down to include only pictures that depicted erotic, fearful or neutral scenes it was clear that the mentioned effects were driven by the erotic images; with participants making slower prosaccades towards erotic images. There was also a clear effect of emotion group when inspecting error rates, with erotic images causing a much higher percentage of errors than fearful images, followed by neutral images. Finally data from experiment four showed a similar pattern to experiments one and two with prosaccades made faster than antisaccades and more errors present in the antisaccade task. An effect of emotion was also found, with the PH pictures causing slower saccades than the remaining groups for both saccade types. This effect was emphasised when the groups were broken down to erotic, fearful and neutral with erotic pictures slowing down saccades made towards or away from them. Error rates for these three groups showed the same trend found in experiment three.

There were three predictions made by the current study. The first was that antisaccades would be more prone to effects of emotional content than prosaccades. Data from experiment 
three demonstrate an effect of emotion for prosaccades, but not antisaccades; which can be attributed to inhibition of return. Findings from experiment four demonstrate similar effects of emotion for both the prosaccade and antisaccade tasks. While experiments three and four show effects of emotional content, neither suggest that antisaccades are more vulnerable to emotional content. The data does propose that even when the individual has processed the semantic information well before the task is known and executed, the influence of that information is independent to spatial orienting and ocular control. This implies that emotional processing and ocular control occur on separate levels.

The study also predicted that high arousal emotional images would have a larger effect than the low arousal images. While this was present in experiments three and four and the PH group being the only group to cause significant interference of saccade initiation, there were no effects found for the NH group. This required a closer look into the differences between these high arousal groups. When the high arousal groups were reduced to erotic pictures from the $\mathrm{PH}$ group and fearful pictures from the $\mathrm{NH}$, it became apparent that the effect was driven by the erotic group alone. This suggests that the erotic pictures are processed differently from the remaining stimuli. There could be two possible explanations for the slowing of eye movements made towards or away from erotic pictures. The first could be the context of the viewing. Because the participant is currently in a university setting and presented with erotic pictures, the setting and the stimuli may produce a conflict, slowing down any responses regarding these images. The slowing of saccades may also be explained with the unique properties of erotic pictures. While erotic pictures have been found to elicit stronger ERP than other positive images (Schupp et al., 2004; van Lankveld \& Smulders, 2008), they have also been found to activate different areas which are not active during viewing of non-erotic images. One such area is the insula (Redouté et al., 2000; Walter et al., 2008); an area also involved in eye movements (Anderson et al., 1994; Haller et al., 2008). As this single area is activated by erotic pictures and eye movements, this may strain the processing capacity of the insula, resulting in the observed slowing down of both pro- and antisaccades and increasing 
errors for this emotion group. The overloading of the insula also explains why erotic images increased latencies for experiments three and four, not experiments one and two.

The final prediction was that participants would use approach and avoidance behaviours when making saccades towards or away from the images. Experiment three and four were the only experiments to show interaction between saccades and emotional pictures. This interaction however does not suggest participants were faster to saccade towards the positive pictures, and slower to saccade away from them. Approach and avoidance behaviours were however observed in the Stormark and Hugdahl $(1996 ; 1999)$ and Mackintosh and Mathews (2003) studies. This could be due to Stormark and Hugdahl $(1996 ; 1999)$ studies using classical conditioning which is a powerful technique used to alter behaviour (in this case invoke avoidant behaviours). While the Mackintosh and Mathews (2003) study had participants choose between a positive picture and a negative picture, allowing participants to always look at an image, opposed to the single choice paradigm used by the current study where participants look at either an image, or an empty space.

The absence of approach and withdrawal behaviours may also be explained through the categorisation of the IAPS. The emotional groups used in the current study were categorised into the $\mathrm{PH}, \mathrm{PL}, \mathrm{NH}, \mathrm{NL}$ and NE groups. These groups are purely based on the valence and arousal ratings of each picture gathered from a number of studies (Lang, Bradley, \& Cuthbert, 1997). However multiple emotions can be found within a single group. For example while the $\mathrm{NH}$ group contains pictures that have scored highly in arousal and have a low valence rating, pictures within this group can also fall into two subgroups: fearful and disgust. While pictures in these groups have similar valence and arousal ratings, the purpose of each emotion is quite different. Susskind and colleagues (2008) demonstrate this through analysis of typical facial expressions that represent each emotion. They found that facial expressions that portray fear involve widening of the eyes and nasal passages. This is to increase the visual field and increase oxygen intake respectively. The facial expression for disgust is portrayed with the opposite facial movements, where eyes and nasal passages are 
scrunched up to limit the amount of visual and olfactory information entering through these two senses. These two behaviours could be likened to pro- and antisaccades, where prosaccades like the fear expression encourage information processing, while antisaccades like the disgust expression discourage information processing. The fact that these two polar opposite emotions can be grouped together using the valence and arousal continuum given by the IAPS suggests that this grouping technique is not suitable for experimental designs like the current study.

Experiments one and two followed the basic methodology of Hallatt's (1978) antisaccade task by presenting the IC 200 ms before the target. While data from these two experiments support findings of previous studies (Hallett, 1978; Ansari, et al., 2008; Godijn \& Kramer, 2007; 2008; Kissler \& Keil, 2008; Nguyen, Mattingley, \& Abel, 2008), emotional content of the target had no effect on pro- or antisaccade latencies. This suggests that the traditional antisaccade task is immune to influence from emotional content. This contradicts findings from other visual paradigms reviewed in the introduction. The free viewing paradigm illustrated how picture content can alter the individuals looking behaviours (Green et al., 2003) and how these behaviours are modulated by the personal coping strategies of that individual (Pflugshaupt et al., 2007). A similar trend was found using the visual search task where a pull of attention towards threatening pictures was observed, resulting in faster search times (Flykt \& Caldara, 2006; Horley, et al., 2003; 2004; Öhman, Flykt, \& Esteves, 2001). This was also found with Nummenmaa and colleagues' (2006) dot-probe study, where attention was attracted towards emotional images when paired with neutral images. While these three paradigms support a bias towards emotional pictures; the antisaccade task used in the current study did not display any effects of emotional content on viewing behaviours. This can be explained through the differences between these studies and the antisaccade task. In the free viewing paradigm images were presented for long intervals, allowing the participant to process the image in great detail. The antisaccade task used in the current study however, presented the image the same time an eye movement is required, not allowing enough time to process the emotional content of the image. The visual search studies used stimuli that were relevant to their participants. Horley and colleagues 
(2003; 2004) use a social phobic population and had faces as their threatening stimuli, while Öhman and colleagues (2001) and Flykt and Caldara (2006) used specific fearful images to match a specific phobic population.

Experiments three and four were created to tease out emotional effects that were found by other visual paradigms, but were not present in experiments one and two. To achieve this, the order of target and IC was reversed with the target presented before the IC (- $200 \mathrm{~ms} \mathrm{SOA}$ ) allowing enough time for the image to be processed in the periphery before the eye movement was initiated. Hallatt's (1978) original antisaccade paradigm relies on the immediate competition of the inhibitory forces of the antisaccade working against the excitatory forces of the reflexive prosaccade. By changing the order of image and IC presentation in experiments three and four, this competition is interrupted. In the new method, the reflexive prosaccade is inhibited regardless of trial type as participants are required to maintain central fixation until the IC is presented and then make the prosaccade or antisaccade. While this limits the comparability to the traditional antisaccade paradigm used in experiments one and two, experiments three and four did provide effects of emotional content. An interaction of saccade and emotional content was found in experiment three, while an effect of emotional content was present in experiment four. As there was no emotional effect present in experiments one and two, data from experiments three and four suggest that emotional content needs to be presented before the saccade direction is known to have an effect on the inhibition or initiation of saccades. In the Kissler and Keil (2008) study a 200 ms SOA was used, this may explain why the study did not find any effect of emotional content on pro- or antisaccades.

The difference between the SOA groups can be explained with both Munoz and Everling (2004) and Findlay and Walker (1996) models. Both models emphasise the competing force between pro- and antisaccades as being the inhibition component. Where prosaccades are reflexive and are initiated with an excitatory signal which occurs with little effort; antisaccades begin with a 
large inhibitory signal to block the reflexive prosaccade. Applying this to the current study, when the IC is presented in experiments one and two, participants are instructed to prepare for either a prosaccade, resulting in excitation of the SC; or an antisaccade, resulting in inhibition of the SC. This means that the participant will have their saccade ready for firing when the appropriate direction is indicated by the placement of the target. This may result in participants relying on the image solely as a temporal and spatial cue, keeping processing of the content to a minimum. As the saccade has already been chosen and prepared before the picture is presented, any effects of emotional content are processed too late to influence the eye movement. In experiment three and four the image is presented first, allowing the emotional content of the image to be processed before the IC is presented. Now the participant is aware of the basic content of the picture and is relying on the IC to indicate which type of saccade is required. As preparation for the saccade has not yet been made, the emotional content of the picture is able to influence the excitation of the prosaccade or the inhibition of the antisaccade, resulting in differences in latencies observed across the emotional groups

While experiments three and four produced emotional effects not present in experiments one and two, three and four also produced different results to each other. Experiment three could be considered as the black sheep of the study due to its inversed direction effect with longer latencies for prosaccades than antisaccades. This could be due to a combination of the experiment design and a phenomenon known as inhibition of return (IOR). The -200 ms SOA and single block tasks allow participant to know which saccade to make and which direction to make it to before the IC is presented, allowing them to pre-plan the appropriate saccade to the correct area. The presentation of the target will attract the participant's exogenous attention, which will then be pulled to the IC. During antisaccade trials attention will move to the opposite side to the image, while during prosaccade trials attention will then return to the image for the saccade to be made. This is where IOR comes into effect. IOR describes the inhibiting force on that acts against the movement of attention towards a recently attended space (Klein, 2000) or object (Tipper, Driver, \& 
Weaver, 1991). Inhibition appears 200 ms after attention has been removed from the object or area and may persist up to $600 \mathrm{~ms}$ after the relocation of attention. As attention returns to the image for prosaccade trials in experiment three, IOR acts to inhibit this movement resulting in extended latencies. During antisaccade trials attention is moved to a new location and therefore immune to IOR, creating shorter latencies than prosaccades.

Inhibition of return was not present in experiments one and two due to the order of the IC and target. As the target was presented second and indicated to the participant that the saccade can be made, attention in the prosaccade trials moves from the IC to the target and remains there for the saccade. IOR was also not present in experiment four, which shares the same method as experiment three; the only differentiating factor between the two experiments is that four has mixed task blocks while experiment three has single task blocks. After the first trial of each single task block in experiment three the participant would know that each subsequent trial in the block will be the same task. This in turn reduces the function of IC no more than a temporal cue. In the mixed tasks blocks in experiment four, participants rely on the IC to indicate the saccade type before they can plan saccade. This creates a more complex task as reflected in the overall increase of saccade latencies. Now the participant has to wait until the IC is presented, discriminate the colour and then interpret this as the appropriate saccade. After these steps have been made, it is now a matter of making a saccade towards a target, or to an empty space. As the latter is harder to perform (Land, 1999), this results in shorter latencies for prosaccades than antisaccades. The abstract relationship between the IC colour and prosaccades also require the participant to rely heavily on endogenous attention (attention that is controlled by the participant) to push their attention to the appropriate area. This type of attention is also immune to effects of IOR (Rafal, Calabresi, Brennan, \& Sciolto, 1989) which may also explain why the inversed direction effect was found in experiment three, but not experiment four. 
Findings from the current study can be used in a number of different settings. In visual studies that employ emotional pictures, data from this study can serve as a warning that erotic pictures will slow down reaction times independent of the experimental manipulation, requiring experimenters to account for the slowing of saccades when using erotic pictures. In a more practical setting, data from the current study should be considered when using of erotic pictures in advertising. While erotic images will attract attention, saccades made towards and away from these images will be delayed. This may be an undesirable effect in some environments, i.e. road side billboards.

The null emotional effects in the current study may be due to the images that were used. If this study were to be replicated, images should be selected and grouped according to the emotion portrayed, not the valence and arousal rating given by the picture system. The reliance on erotic pictures to produce the effects in the current study could be countered by using stimuli that have more relevance to the population tested on as used in the visual search paradigms (Flykt \& Caldara, 2006; Horley, et al., 2003; 2004; Öhman, Flykt, \& Esteves, 2001). This could be done by using fearful pictures and phobic participants, or manipulating the testing environment to make images more appetitive. This could be done by lowering the temperature in the lab and present pictures of heaters or jackets, or snow and cold weather. Having participants fast for a period of time before the experiment and then present images of food would also increase the appeal of the images. While these changes will make the images more relevant to the participant they may also tap into core survival needs, (i.e. warmth, food) which may produce the approach and withdrawal behaviours predicted by the current study.

In conclusion, does emotional content play a role in eye movements? Where previous visual studies found that emotional content attracts attention, using the antisaccade paradigm the current study found that when the image is presented simultaneously to the initiation of the saccade, that movement will not be influenced by the emotional content of the image. When the image is 
presented $200 \mathrm{~ms}$ before the saccade is initiated however, emotional content can affect the speed of saccade activation. While there was an interaction of saccade type and emotional group, the inconsistency of this interaction across experiments three and four imply that the processing of emotional content occurs on a different level to that of ocular control. Effects of emotional content in both studies were also driven solely by erotic pictures as demonstrated by the decrease in latencies for prosaccades in experiment three and both prosaccades and antisaccades in experiment four. This raises the question for future research of whether the effect of emotional content found in experiments three and four are due to the actual emotion portrayed by the image, or if this interference was due to the unique properties of erotic pictures. 


\section{References}

Adobe Photoshop version 10.0 [Computer software] (1990 - 2007). San Jose, California: Adobe Systems Incorporated.

Anderson, T. J., Jenkins, I. H., Brooks, D. J., Hawken, M., Frackowiak, R. S., \& Kennard, C. (1994). Cortical control of saccades and fixation in man A PET study. Brain , 117 (5), 1073-1074.

Ansari, T. L., Derakshan, N., \& Richards, A. (2008). Effects of anxiety on task switching: Evidence from the mixed antisaccade task. Cognitive, Affective and Behavioral Neuroscience , 8 (3), 229238.

Broomfield, N. M. \& Turpin, G. (2005). Covert and overt attention in trait anxiety: a cognitive psychophysiological analysis. Biological Psychology , 68, 179-200.

Campanella, S. \& Guerit, J. M. (2009). How clinical neurophysiology may contribute to the understanding of a psychiatric disease such as schizophrenia. Clinical Neurophysiology, 39 (1), 31-39.

Carpenter, R. H. S.(1999). A neural mechanism that randomises behaviour. Journal of Consciousness Studies , $6(1), 13-22$.

Chan, F., Armstrong, I. T., Pari, G., Riopelle, R. J., \& Munoz, D. P. (2005). Deficits in saccadic eyemovement control in Parkinson's disease. Neuropsychologia , 43 (5), 784-796.

Curtis, C. E. \& D'Esposito, M. (2003). Success and failure suppressing reflexive behaviour. Journal of Cognitive Neuroscience, 15 (3), 409-418.

Davidson, R. J. (1998). Affective style and affective disorders: Perpectives from affective neuroscience. Cognition and Emotion , 12 (3), 307-330. 
Findlay, J. M. \& Walker, R. (1999). A model of saccade generation based on parallel processing and competitive inhibition. Behavioral and Brain Sciences, 22, 661-721.

Flykt, A. \& Caldara, R. (2006). Tracking fear in snake and spider fearful participants during a visual search: a multi-response domain study. Cognition and Emotion , 20 (8), 1075-1091.

Fox, E. (2002). Processing emotional facial expressions:The role of anxiety and awareness. Cognitive, Affective and Behavioural Neuroscience , 2 (1), 52-63.

Gerdes, A. B., Pauli, P., \& Alpers, G. W. (2009). Toward and away from spiders: eye movements in spider-fearful participants. Journal of Neural Transmission , 116, 725-733.

Godijn, R. \& Theeuwes, J. (2003). Parallel allocation of attention prior to the execution of saccade sequences. Journal of Experimental Psychology: Human Perception and Performance , 29 (5), 882-896.

Godijn, R. \& Kramer, A. F. (2007). Antisaccade costs with static and dynamic targets. Perception and Psychophysics, 69 (5), 802-815.

Godijn, R. \& Kramer, A. F. (2008). Effects of attentional demands on the antisaccade cost. Perception and Psychophysics, 70 (5), 795-806.

Gray, J. A. (1990). Brain systems that mediate both emotion and cognition. Cognition and Emotion , 4 (3), 269-288.

Green, M. J., Williams, L. M., \& Davidson, D. (2003). In the face of danger: specific viewing strategies for facial expressions of threat? Cognition and Emotion , 17 (5), 779-786.

Haller, S., Fasler, D., Ohlendorf, S., Radue, E., \& Greenlee, M. W. (2008). Neural activation associated with the corrective saccades during tasks with fixation, pursuit and saccades. Experimental Brain Research, 184, 83-94. 
Hallett, P. E. (1978). Primary and secondary saccades defined by instructions. Vision Research, 18 (10), 1279-1296.

Hanes, D. P., Patterson, W. F., \& Schall, J. D. (1998). Role of frontal eye fields in countermanding saccades: Visual, movement, and fixation activity. Journal of Neurophysiology, 79 (2), 817834.

Henderson, J. M. (2003). Human gaze control during real-world scene perception. Trends in Cognitive Sciences , 7 (11), 498-504.

Henderson, J. M., Weeks, P. A., \& Hollingworth, A. (1999). The effects of semantic inconsistency on eye movements during complex scene viewing. Journal of Experimental Psychology: Human Perception and Performance , 25 (1), 210-228.

Hester, R. \& Garavan, H. (2005). Working memory and executive function: The influence of content and load on the control of attention. Memory \& Cognition, 33 (2), 221-233.

Horley, K., Williams, L. M., Gonsalves, C., \& Gordon, E. (2003). Social phobics do not see eye to eye: A visual scanpath study of emotional expression processing. Anxiety Disorders , 17, 33-44.

Horley, K., Williams, L. M., Gonsalvez, C., \& Gordon, E. (2004). Face to face: Visual scanpath evidence for abnormal processing of facial expressions in social phobia. Psychiatry Research, 127, 4353.

Kissler, J. \& Keil, A. (2008). Look-don't look! How emotional picture affect pro- and anti-saccades. Experimental Brain Research, 188, 215-222.

Klein, R. M. (2000). Inhibition of Return. Trends in Cognitive Science , 4 (4), 138-137.

Land, M. F. (1999). Motion and vision: Why animals move their eyes. Journal of Comparative Physiology , 185, 341-352. 
Lang, P. J., Bradley, M. M., \& Cuthbert, B. N. (1997). International Affective Picture System (IAPS): Technical Manual and Affective Ratings. National Institute of Mental Health.

LeDoux, J. E. (2000). Emotion circuits in the brain. Annual Review of Neuroscience , 23, 155-184.

Mackintosh, B. \& Mathews, A. (2003). Don't look now: Attentional avoidance of emotionally valenced cues. Cognition and Emotion , 17 (4), 623-646.

MacLeod, C., Mathews, A. M., \& Tata P. (1986). Attentional bias in emotional disorders. Journal of Abnormal Psychology, 95, 15-20.

Martin, L. F., Hall, M., Ross, R. G., \& Zerbe, G. (2007). Physiology of Schizophrenia, Bipolar Disorder, and Schizoaffective Disorder. The American Journal of Psychiatry, 164 (12), 1900-1906.

Martinez-Trujillo, J. C., Wang, H., \& Crawford, J. D. (2003). Electrical stimulation of the supplementary eye fields in the head-free macaque envokes kinematically normal gaze shift. Journal Neurophysiology , 89 (6), 2961-2974.

McNamee, R. L., Dunfee, K. L., Luna, B., Clark, D. B., Eddy, W. F., \& Tarter, R. E. (2008). Brain activation, response inhibition, and increased risk for substance use disorder. Alcoholism: Clinical and Experimental Research. , 32 (2), 405-413.

Müller, M. M., Anderson, S. K., \& Keil, A. (2008). Time course competition for visual processing resources between emotional pictures and foreground task. Cerebral Cortex, 5, 1892-1899.

Munoz, D. P. \& Everling, S. (2004). Look away: The anti-saccade task and the voluntary control of eye movement. Nature , 5, 218-228.

Nguyen, H. N., Mattingley, J. B., \& Abel, L. A. (2008). Extraversion degrades performance on the antisaccade task. Brain Research , 1231, 81-85. 
Nummenmaa, L., Hyönä, J., \& Calvo, M. G. (2006). Eye movement assessment of selective attentional capture by emotional pictures. Emotion , 6 (2), 257-268.

Öhman, A., Flykt, A., \& Esteves, F. (2001). Emotion drives attention: detecting the snake in the grass. Journal of Experimental Psychology: General , 130 (3), 466-478.

Pflugshaupt, T., Mosimann, U. P., Schmitt, W. J., von Wartburg, R., Wurtz, P., Luthi, M., et al. (2007). To look or not to look at threat? Scanpath differences within a group of spider phobics. Journal of Anxiety Disorders , 21, 353-366.

Posner, M. I., Synder, C. R., \& Davidson, B. J. (1980). Attention and the detection of signals. Journal of Experimental Psychology, 109 (2), 160-174.

Rafal, R. D., Calabresi, P. A., Brennan, C. W., \& Sciolto, T. K. (1989). Saccade preparation inhibits reorienting to recently attented locations. Journal of Experimental Psychology: Human perception and Performance, 15 (4), 673-685.

Rayner, K., Smith, T. J., Malcolm, G. L., \& Henderson, J. M. (2009). Eye movements and visual encoding during scene perception. Psychological Science , 20 (1), 6-10.

Redouté, J., Stoléru, S., Grégoire, M., Costes, N., Cinotti, L., Lavenne, F., et al. (2000). Brain processing of visual sexual stimuli in human males. Human Brain Mapping , 11, 162.177.

Reuter, B. \& Kathmann, N. (2004). Using saccade tasks as a tool to analyze executive dysfunction in schizophrenia. Acta Psychologica, 115, 255-269.

Ribeiro, R. L. \& Pompéia, S., Bueno, O. F. A. (2005). Comparison of Brazilian and American norms for the International Affective Picture System (IAPS). Revista Brasileira de Psiquiatria , 27 (3), 208-215.

Rozenkrants, B. \& Polich, J. (2008). Affective ERP processing in a visual oddball task: arousal, valence, and gender. Clinical Neurophysiology , 119 (10), 2260-2265. 
Schupp, H. T., Cuthbert, B. N., Bradley, M. M., Hillman, C. H., Hamm, A. O., \& Lang, P. J. (2004). Brain processes in emotional perception: Motivated attention. Cognition and Emotion , 18 (5), 593-611.

Segraves, M. A. (1992). Activiy of monkey frontal eye field neurons projecting to oculomotor regions of the pons. Journal of Neurophysiology, 68, 1967-1985.

Smith, O. A. \& DeVito, J. L. (1984). Central neural integration for the control of autonomic responses associated with emotion. Annual Review of Neuroscience , 7, 43-65.

Stormark, K. M. \& Hugdahl, K. (1996). Peripheral cuing of covert spatial attention before and after emotional condition of the cue. International Journal of Neuroscience, 86 (3-4), 225-240.

Stormark, K. M., Hugdahl, K. \& Posner M. I., (1999). Emotional modulation of attention orienting: A classical conditioning study. Scandinavian Journal of Psychology, (40), 91-99

Susskind, J. M., Lee, D. H., Cusi, A., Feiman, R., Grabski, W., \& Anderson, A. K. (2008). Expressing fear enhances sensory acquisition. Nature Neuroscience , 11 (7), 843-850.

Tipper, S. P., Driver, J., \& Weaver, B. (1991). Object-centred inhibition of return of visual attention. The Quarterly Journal of Experimental Psychology A: Human Experimental Psychology, 43A (2), 289-298.

Torralba, A., Oliva, A., Castelhano, M. S., \& Henderson, J. M. (2006). Contextual guidance of eye movements and attention in the real-world scenes: The role of global features in object search. Psychological Review, 113 (4), 766-786.

van Lankveld, J. \& Smulders, F. (2008). The effect of visual sexual content on the event-related potential. Biological Psychology , 79, 200-208.

Verschuer, B., Crombez, G., \& Koster, E. (2001). The International Affective Picture System: A Flemish validation study. Psychologica Belgica , 41 (4), 205-217. 
Vuilleumier, P. \& Schwartz, S. (2001). Beware and be aware: Capture of spatial attention by fearrelated stimuli in neglect. Motivation, Emotion, Feeding, Drinking , 12 (6), 1119-1122.

Walter, M., Bermpohl, F., Mouras, H., Schiltz, K., Tempelmann, C., Rotte, M., et al. (2008). Distinguishing specific sexual and general emotional effects in fMRI - Subcortical and cortical arousal during erotic picture viewing. Neurolmage , 40, 1482-1494.

Yarbus, A. L. (1967). Eye Movements and Vision. New York: Plenum Press.

Yuxia, H. \& Yuejia, L. (2004). Native assessment of International Affective Picture System. Chinese Mental Health, 18 (9), 631-634. 


\begin{tabular}{|c|c|c|c|c|c|c|c|c|c|}
\hline \multicolumn{2}{|c|}{ Positive High } & \multicolumn{2}{|c|}{ Positive Low } & \multicolumn{2}{|c|}{ Negative High } & \multicolumn{2}{|c|}{ Negative Low } & \multicolumn{2}{|c|}{ Neutral } \\
\hline 1650 & 4689 & 1340 & 5994 & 1052 & 6370 & 2141 & 9265 & 2200 & 7002 \\
\hline 4607 & 4690 & 1540 & 7250 & 1201 & 6415 & 2276 & 9290 & 2214 & 7009 \\
\hline 4608 & 4810 & 1590 & 7282 & 1525 & 6530 & 2455 & 9320 & 2385 & 7034 \\
\hline 4611 & 5470 & 1660 & 7350 & 2683 & 6570 & 2700 & 9330 & 2393 & 7036 \\
\hline 4652 & 5626 & 1731 & 7390 & 2688 & 6821 & 2715 & 9340 & 2493 & 7037 \\
\hline 4656 & 5629 & 2224 & 7410 & 2981 & 6830 & 2750 & 9342 & 2514 & 7038 \\
\hline 4658 & 8034 & 2344 & 7430 & 3150 & 6834 & 2900 & 9415 & 2516 & 7130 \\
\hline 4659 & 8116 & 2352 & 7470 & 5971 & 8485 & 3181 & 9417 & 2595 & 7160 \\
\hline 4660 & 8161 & 2391 & 7480 & 6200 & 9050 & 3300 & 9421 & 2749 & 7161 \\
\hline 4664 & 8178 & 2655 & 7481 & 6210 & 9250 & 4621 & 9432 & 2880 & 7170 \\
\hline 4670 & 8180 & 4150 & 8033 & 6243 & 9300 & 6311 & 9435 & 2890 & 7179 \\
\hline 4676 & 8186 & 4603 & 8120 & 6250 & 9400 & 6561 & 9452 & 5510 & 7184 \\
\hline 4677 & 8193 & 4614 & 8162 & 6300 & 9600 & 7361 & 9470 & 5531 & 7207 \\
\hline 4680 & 8300 & 5820 & 8350 & 6312 & 9620 & 9007 & 9471 & 5532 & 7233 \\
\hline 4681 & 8400 & 5849 & 8461 & 6315 & 9622 & 9041 & 9561 & 5534 & 7235 \\
\hline 4687 & 8490 & 5890 & 8510 & 6360 & 9630 & 9180 & 9830 & 6150 & 9070 \\
\hline
\end{tabular}

Table 1: IAPS images selected for each emotional group. 


\begin{tabular}{lllllll}
\multicolumn{5}{c}{ Valence } & \multicolumn{5}{c}{ Arousal } \\
\hline & Minimum & Maximum & $\mathrm{M}(\mathrm{SD})$ & Minimum & Maximum & $\mathrm{M}(\mathrm{SD})$ \\
Positive High & 6.58 & 7.40 & $6.89(0.21)_{\mathrm{a}}$ & 6.04 & 6.62 & $6.33(0.21)_{\mathrm{c}}$ \\
Positive Low & 6.53 & 7.33 & $6.98(0.25)_{\mathrm{a}}$ & 4.57 & 4.89 & $4.73(0.10)_{\mathrm{d}}$ \\
Negative High & 2.33 & 3.10 & $2.69(0.24)_{\mathrm{b}}$ & 5.99 & 6.54 & $6.28(0.16)_{\mathrm{c}}$ \\
Negative Low & 2.44 & 3.19 & $2.83(0.25)_{\mathrm{b}}$ & 4.40 & 5.03 & $4.78(0.21)_{\mathrm{d}}$ \\
Neutral & 4.14 & 5.14 & $4.94(0.33)$ & 3.08 & 3.41 & $3.24(0.12)$ \\
\hline
\end{tabular}

Table 2: Valence and arousal boundaries for the each emotion group. Items that have the same subscript indicate no significant difference ( $p>0.5)$ 


\begin{tabular}{|c|c|c|c|c|c|c|c|c|}
\hline & \multicolumn{2}{|c|}{ Experiment 1} & \multicolumn{2}{|c|}{ Experiment 2} & \multicolumn{2}{|c|}{ Experiment 3} & \multicolumn{2}{|c|}{ Experiment 4} \\
\hline & Mean & SD & Mean & SD & Mean & SD & Mean & SD \\
\hline Prosaccade & 163.38 & 49.52 & 222.61 & 71.24 & 300.14 & 89.16 & 362.37 & 78.42 \\
\hline Positive High & 163.45 & 49.32 & 224.92 & 69.76 & 311.74 & 85.32 & 370.52 & 71.31 \\
\hline Positive Low & 164.34 & 48.87 & 218.53 & 69.48 & 303.78 & 91.54 & 365.24 & 93.03 \\
\hline Negative High & 162.91 & 51.97 & 223.51 & 81.53 & 291.49 & 85.64 & 362.63 & 71.44 \\
\hline Negative Low & 163.45 & 53.51 & 226.17 & 69.61 & 295.42 & 90.29 & 355.19 & 80.29 \\
\hline Neutral & 162.76 & 45.67 & 219.94 & 71.25 & 298.26 & 94.97 & 358.28 & 76.42 \\
\hline Antisaccade & 229.77 & 54.61 & 276.18 & 78.50 & 266.06 & 83.39 & 408.34 & 95.51 \\
\hline Positive High & 299.01 & 56.00 & 280.89 & 81.09 & 260.91 & 86.43 & 436.23 & 129.80 \\
\hline Positive Low & 228.74 & 45.21 & 269.38 & 77.67 & 274.08 & 84.47 & 394.71 & 73.11 \\
\hline Negative High & 231.53 & 58.48 & 275.50 & 74.44 & 266.41 & 83.31 & 404.32 & 86.71 \\
\hline Negative Low & 227.27 & 58.43 & 272.32 & 75.33 & 269.50 & 86.52 & 406.03 & 90.11 \\
\hline Neutral & 232.31 & 55.98 & 282.83 & 85.90 & 259.19 & 78.60 & 400.42 & 86.49 \\
\hline
\end{tabular}

Table 3: Means and standard deviations for reaction times $(\mathrm{ms})$ for the five emotion groups across the two saccade conditions. 


\begin{tabular}{|c|c|c|c|c|c|c|c|c|}
\hline & \multicolumn{2}{|c|}{ Experiment 1} & \multicolumn{2}{|c|}{ Experiment 2} & \multicolumn{2}{|c|}{ Experiment 3} & \multicolumn{2}{|c|}{ Experiment 4} \\
\hline & Mean & SD & Mean & SD & Mean & SD & Mean & SD \\
\hline Prosaccade & $6.25 \%$ & $6.59 \%$ & $4.09 \%$ & $5.43 \%$ & $13.33 \%$ & $11.05 \%$ & $11.30 \%$ & $11.91 \%$ \\
\hline Positive High & $7.42 \%$ & $7.25 \%$ & $5.47 \%$ & $5.41 \%$ & $12.59 \%$ & $10.52 \%$ & $12.63 \%$ & $12.40 \%$ \\
\hline Positive Low & $6.51 \%$ & $6.82 \%$ & $3.26 \%$ & $4.99 \%$ & $13.52 \%$ & $11.99 \%$ & $10.55 \%$ & $10.33 \%$ \\
\hline Negative High & $5.60 \%$ & $5.66 \%$ & $3.13 \%$ & $4.82 \%$ & $14.15 \%$ & $10.85 \%$ & $11.72 \%$ & $12.48 \%$ \\
\hline Negative Low & $5.73 \%$ & $6.55 \%$ & $4.43 \%$ & $6.69 \%$ & $11.53 \%$ & $10.87 \%$ & $10.68 \%$ & $12.02 \%$ \\
\hline Neutral & $5.99 \%$ & $6.69 \%$ & $4.17 \%$ & $4.88 \%$ & $14.87 \%$ & $11.10 \%$ & $10.94 \%$ & $12.53 \%$ \\
\hline Antisaccade & $12.92 \%$ & $10.81 \%$ & $18.88 \%$ & $15.27 \%$ & $11.72 \%$ & $13.41 \%$ & $15.73 \%$ & $14.12 \%$ \\
\hline Positive High & $13.54 \%$ & $10.66 \%$ & $18.23 \%$ & $16.80 \%$ & $12.61 \%$ & $15.02 \%$ & $14.32 \%$ & $11.60 \%$ \\
\hline Positive Low & $12.24 \%$ & $10.86 \%$ & $19.53 \%$ & $14.62 \%$ & $11.81 \%$ & $11.81 \%$ & $18.62 \%$ & $16.23 \%$ \\
\hline Negative High & $13.28 \%$ & $9.26 \%$ & $17.06 \%$ & $12.42 \%$ & $12.48 \%$ & $12.48 \%$ & $15.76 \%$ & $14.59 \%$ \\
\hline Negative Low & $13.67 \%$ & $13.38 \%$ & $20.31 \%$ & $16.18 \%$ & $11.07 \%$ & $11.07 \%$ & $16.41 \%$ & $14.04 \%$ \\
\hline Neutral & $11.85 \%$ & $9.75 \%$ & $19.27 \%$ & $16.35 \%$ & $10.66 \%$ & $10.66 \%$ & $13.54 \%$ & $13.79 \%$ \\
\hline
\end{tabular}

Table 4: Means and standard deviations for error rates of each emotion group across the two saccade conditions. 


\begin{tabular}{|c|c|c|c|c|c|c|c|c|}
\hline & \multicolumn{4}{|c|}{ Experiment 3} & \multicolumn{4}{|c|}{ Experiment 4} \\
\hline & \multicolumn{2}{|c|}{ RT } & \multicolumn{2}{|c|}{ Errors } & \multicolumn{2}{|c|}{ RT } & \multicolumn{2}{|c|}{ Errors } \\
\hline & $M$ & SD & $\mathrm{M}$ & SD & $M$ & SD & $\mathrm{M}$ & SD \\
\hline \multicolumn{9}{|c|}{ Prosaccade } \\
\hline Erotic & 327.91 & 170.95 & $50.39 \%$ & $12.26 \%$ & 389.14 & 71.15 & $52.23 \%$ & $10.41 \%$ \\
\hline Fear & 287.07 & 154.60 & $33.75 \%$ & $15.24 \%$ & 359.25 & 68.37 & $27.60 \%$ & $10.16 \%$ \\
\hline Neutral & 301.52 & 161.25 & $14.97 \%$ & $11.15 \%$ & 358.28 & 73.06 & $11.00 \%$ & $12.67 \%$ \\
\hline
\end{tabular}

\section{Antisaccade}

$\begin{array}{lcccccccc}\text { Erotic } & 263.56 & 147.07 & 52.73 \% & 10.31 \% & 440.33 & 111.64 & 52.04 \% & 10.12 \% \\ \text { Fear } & 266.34 & 144.17 & 26.30 \% & 14.81 \% & 400.30 & 82.29 & 32.08 \% & 12.73 \% \\ \text { Neutral } & 259.19 & 138.62 & 10.68 \% & 13.27 \% & 399.77 & 82.59 & 13.67 \% & 13.39 \%\end{array}$

Table 5: Mean and standard deviations for reaction times and error rates for erotic, fearful and neutral pictures across each saccade condition for experiment 3 and 4. 


\begin{tabular}{lllll} 
& \multicolumn{2}{c}{ Experiment 3 } & \multicolumn{2}{c}{ Experiment 4} \\
& Mean & SD & Mean & SD \\
& & & & \\
\hline Prosaccade & & & & \\
Positive High & -13.48 & 25.56 & -12.23 & 23.91 \\
Positive Low & -5.52 & 27.67 & -6.96 & 43.80 \\
Negative High & 6.77 & 28.81 & -4.35 & 30.93 \\
Negative Low & 2.85 & 36.85 & 3.10 & 25.26
\end{tabular}

\section{Antisaccade}

$\begin{array}{lllll}\text { Positive High } & -1.72 & 23.04 & -35.82 & 68.61 \\ \text { Positive Low } & -14.89 & 31.06 & 5.70 & 32.14 \\ \text { Negative High } & -7.22 & 27.28 & -3.90 & 40.65 \\ \text { Negative Low } & -10.31 & 23.49 & -5.61 & 30.94\end{array}$

Table 6: Means and standard deviations for saccade facilitation of each emotional image group for experiments 3 and 4. Positive numbers indicate facilitation, negative number indicate inhibition. 


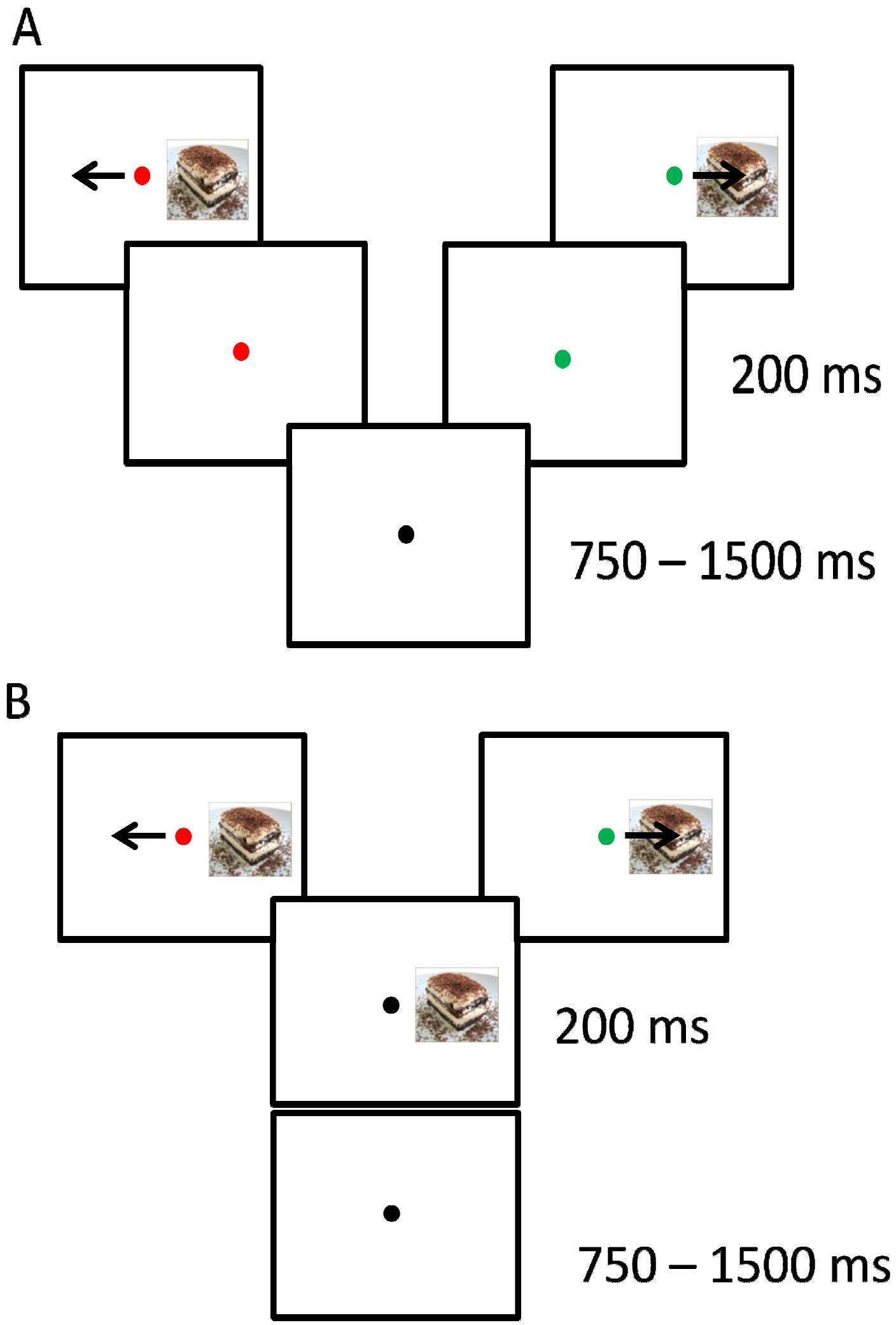

Diagram 1: Sequence of events in experiment 1, $2(A)$, and 3, 4 (B) 


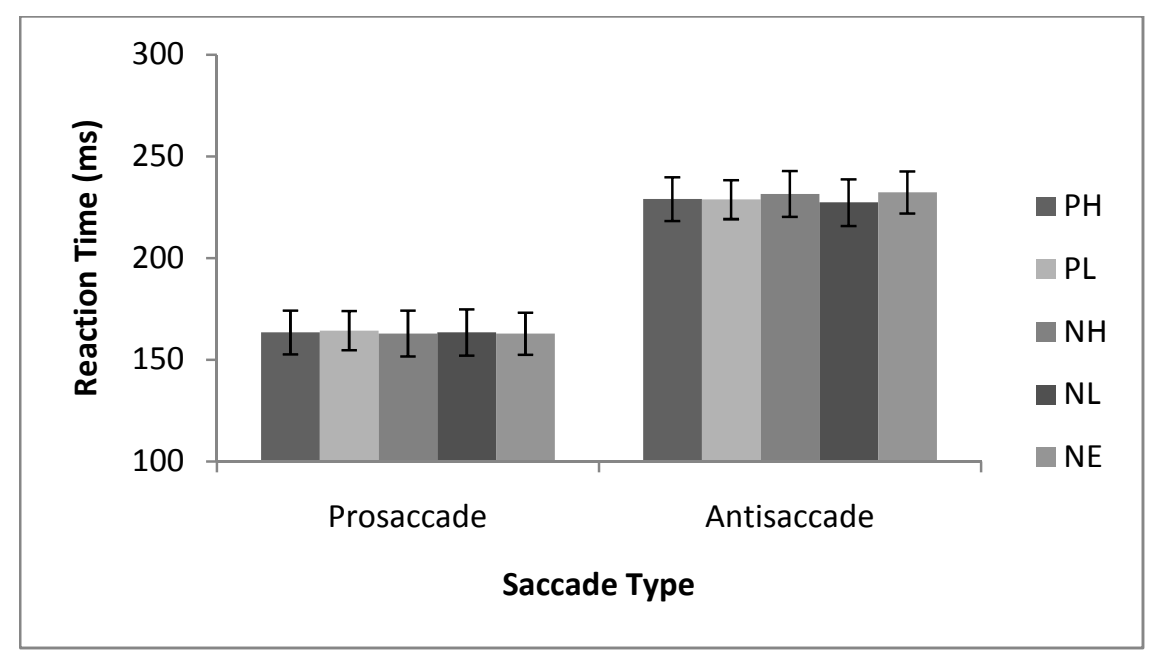

Figure 1: Reaction times for experiment 1. Prosaccades were made significantly faster than antisaccades, but there were no differences between emotion types. (PH: Positive valence, high arousal; $P L=$ Positive valence, low arousal; $N H=$ Negative valence, high arousal; $N L=$ Negative valence, low arousal; $N E=$ Neutral)

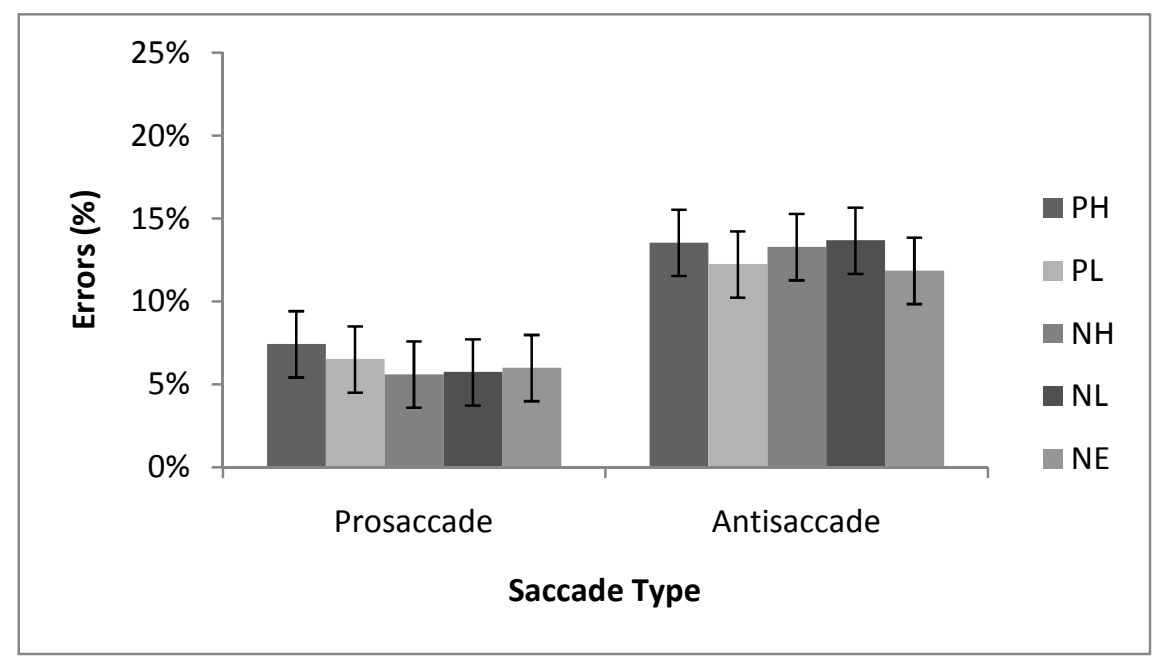

Figure 2: Error rates for experiment 1. There were significantly more errors made in the antisaccade condition than in the prosaccade condition, but no differences across emotion types. (PH: Positive valence, high arousal; $P L=$ Positive valence, low arousal; $N H=$ Negative valence, high arousal; $N L=$ Negative valence, low arousal; NE = Neutral) 


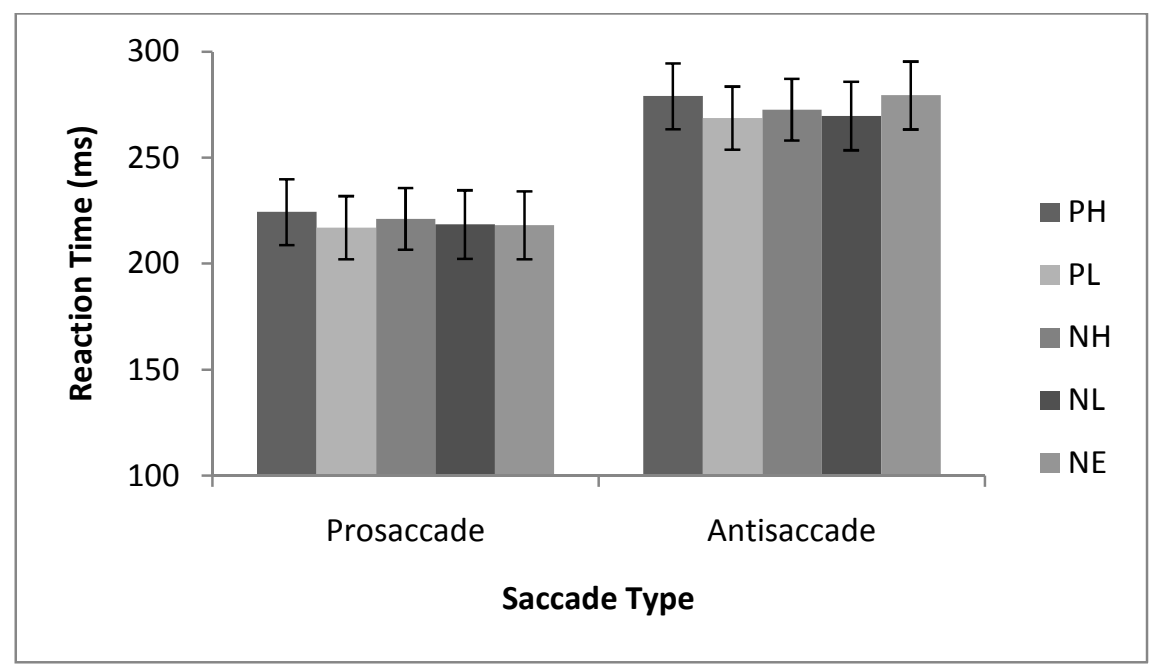

Figure 3: Reaction times for experiment 2. Prosaccades were made significantly faster than antisaccade, but no differences across emotion types. (PH: Positive valence, high arousal; $P L=$ Positive valence, low arousal; $N H=$ Negative valence, high arousal; $N L=$ Negative valence, low arousal; $N E=$ Neutral)

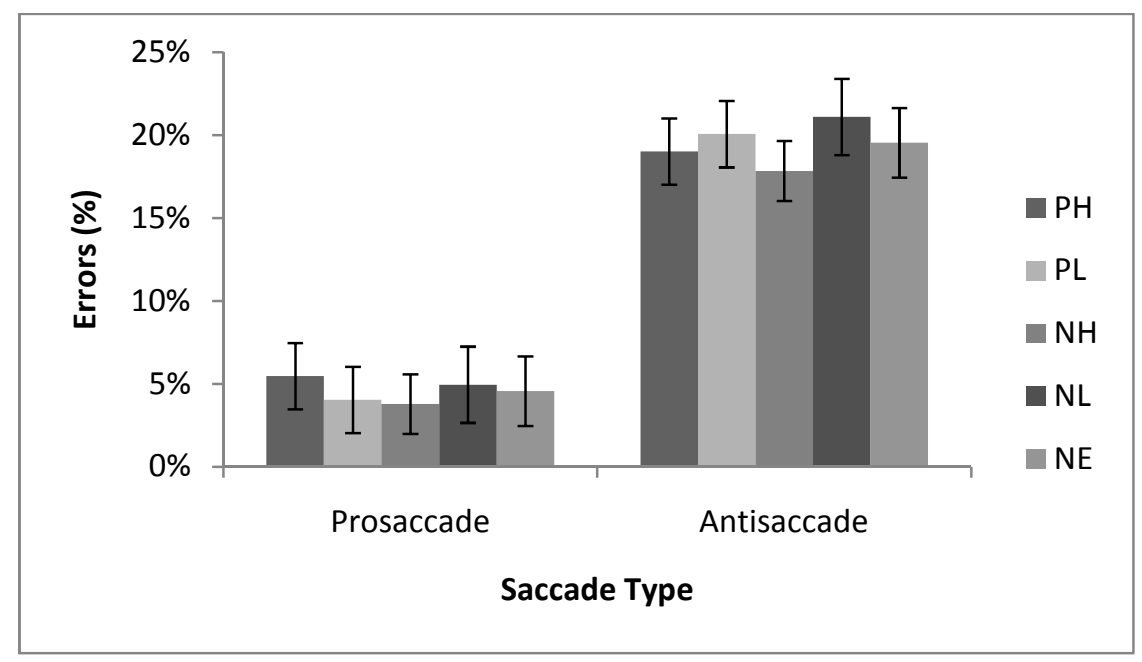

Figure 4: Errors made for experiment 2. There were significantly more errors made in the antisaccade task than the prosaccade task. There were no differences across emotion types. (PH: Positive valence, high arousal; $P L=$ Positive valence, low arousal; $N H=$ Negative valence, high arousal; $N L=$ Negative valence, low arousal; $N E=$ Neutral) 


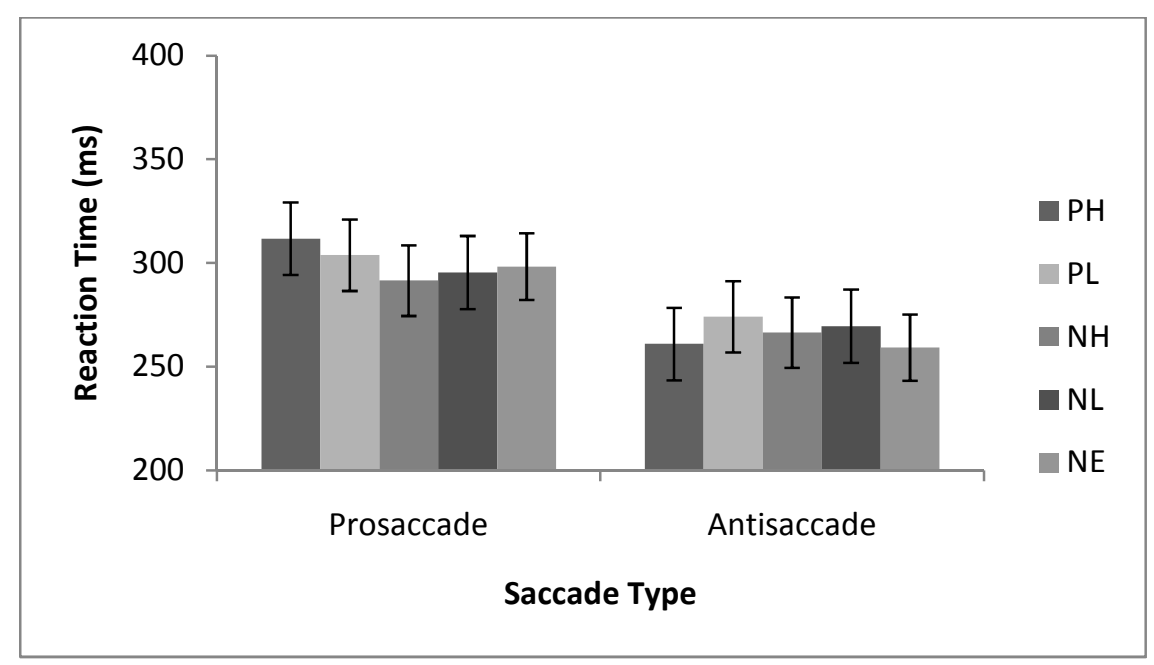

Figure 5: Reaction times for experiment 3. Antisaccades were made significantly faster than prosaccades. There was also an interaction between saccade type and emotion type, which was driven by the positive valence, high arousal group. (PH: Positive valence, high arousal; $P L=$ Positive valence, low arousal; $N H=$ Negative valence, high arousal; $N L=$ Negative valence, low arousal; NE = Neutral)

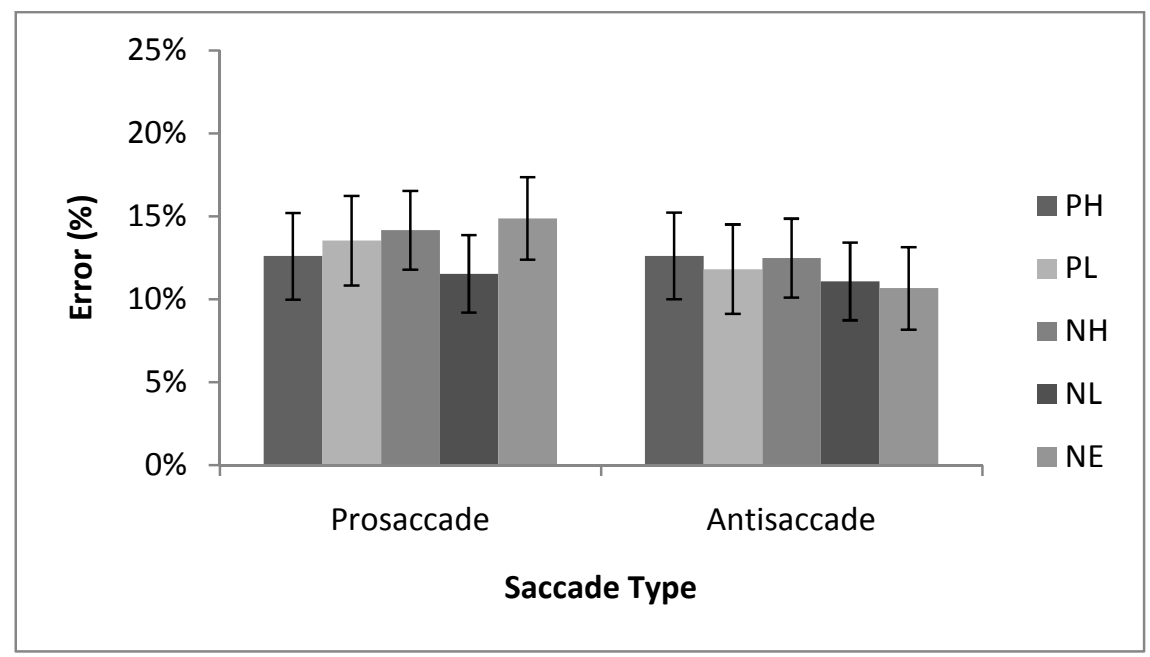

Figure 6: Error rates for experiment 3; no differences were found between saccade or emotion type. (PH: Positive valence, high arousal; $P L=$ Positive valence, low arousal; $\mathrm{NH}=$ Negative valence, high arousal; $N L=$ Negative valence, low arousal; $N E=$ Neutral) 


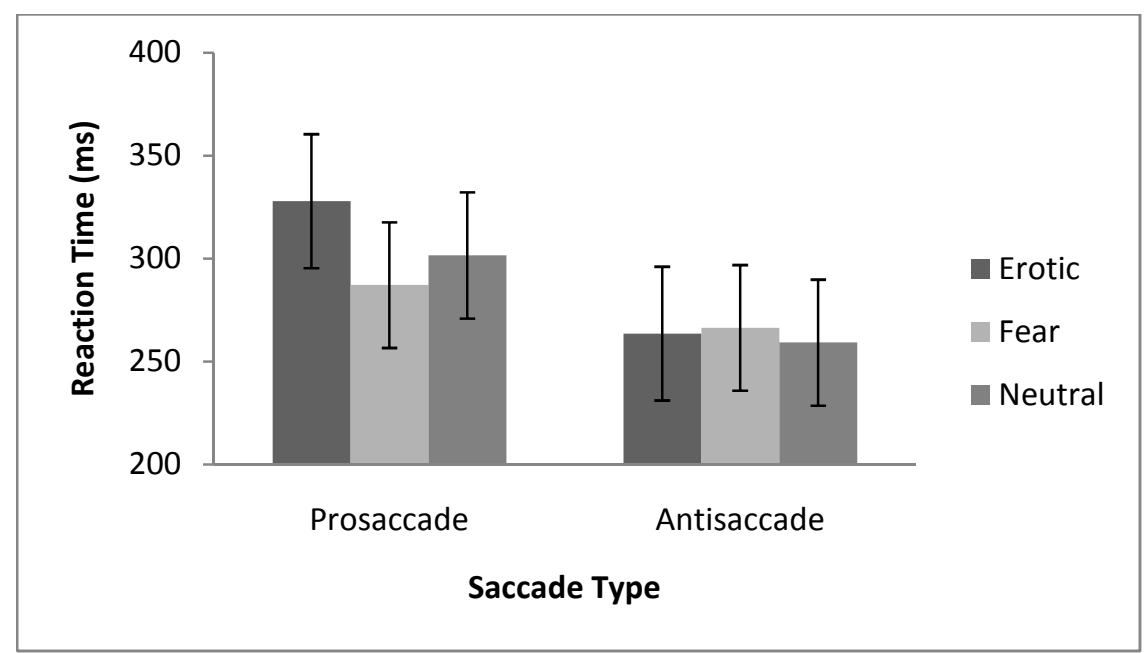

Figure 7: Reaction times for erotic, fearful and neutral pictures in experiment 3; prosaccades took significantly longer to make than antisaccades. Reaction times for erotic pictures were also longer than the remaining two groups in the prosaccade.

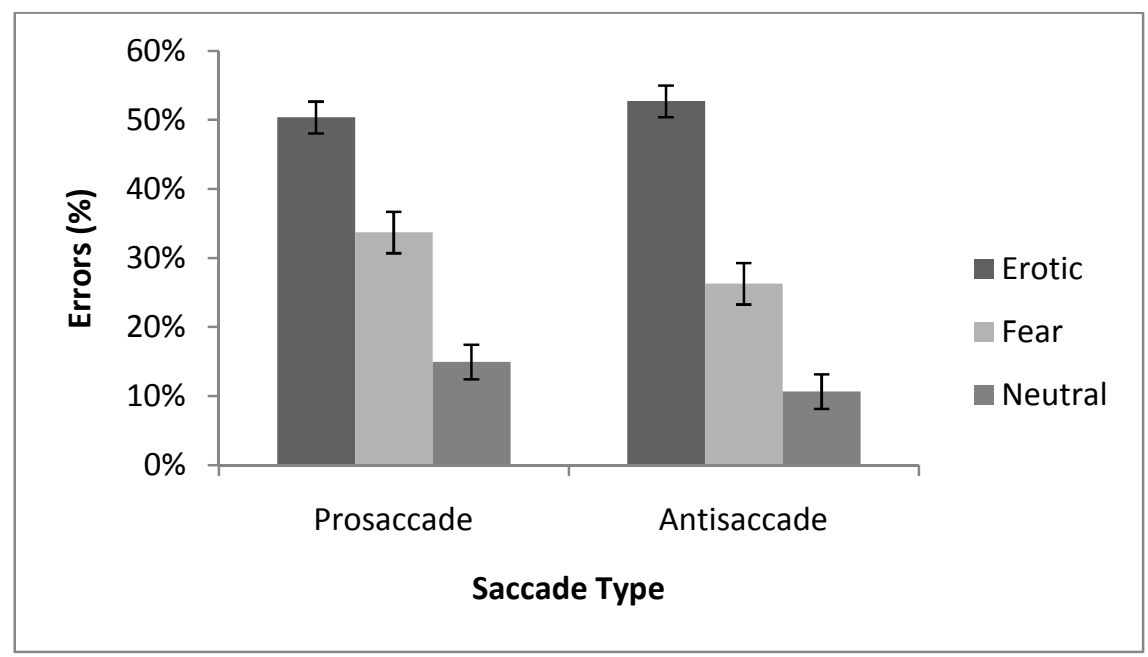

Figure 8: Error rates for erotic, fearful and neutral pictures in experiment 3. There were no differences between the two saccade tasks; however there were significantly more errors for erotic pictures than the remaining two groups. Fearful pictures also had significantly more errors than neutral pictures. 


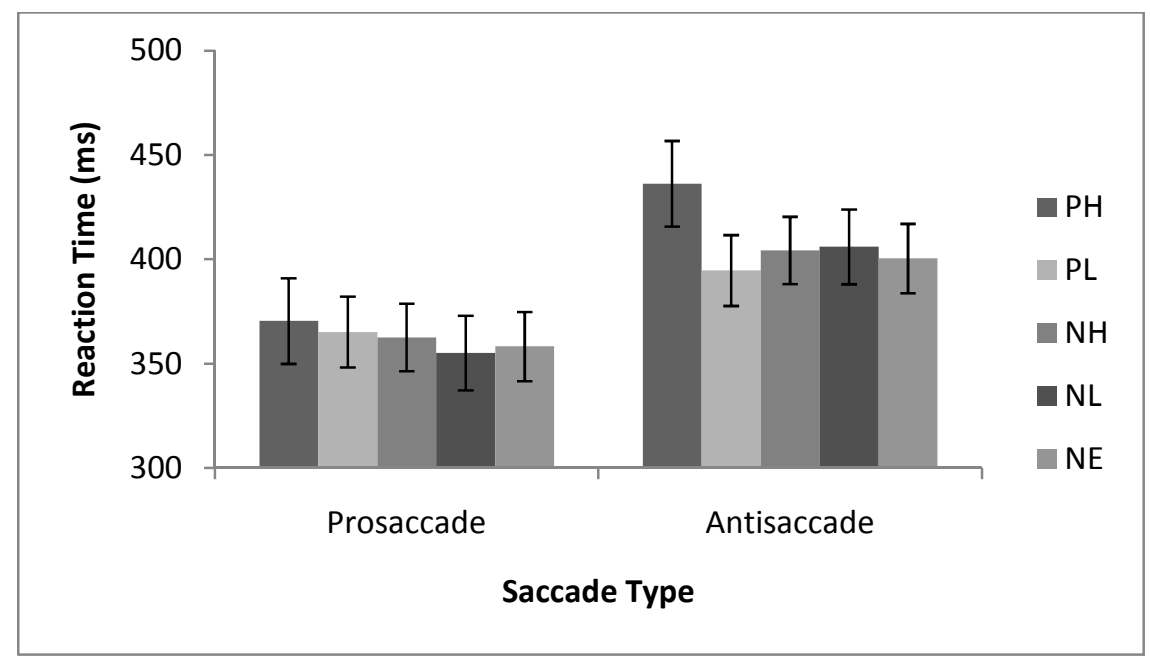

Figure 9: Reaction times for experiment 4; prosaccades were made significantly faster than antisaccades. There was an effect of emotion type, which was found to be driven by the positive high group. (PH: Positive valence, high arousal; $P L=$ Positive valence, low arousal; $N H=$ Negative valence, high arousal; $N L=$ Negative valence, low arousal; $N E=$ Neutral)

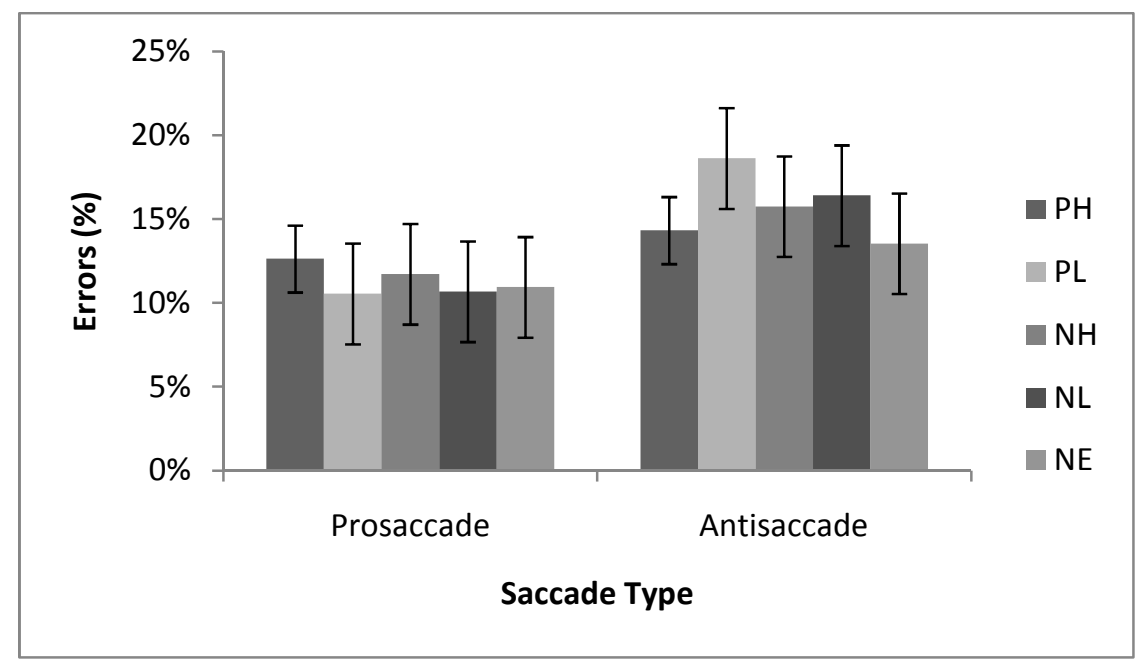

Figure 10: Error rates for experiment 4. There were significantly more errors made for the antisaccade task, but no significant effect of emotion type. (PH: Positive valence, high arousal; $P L=$ Positive valence, low arousal; $N H=$ Negative valence, high arousal; $N L=$ Negative valence, low arousal; NE = Neutral) 


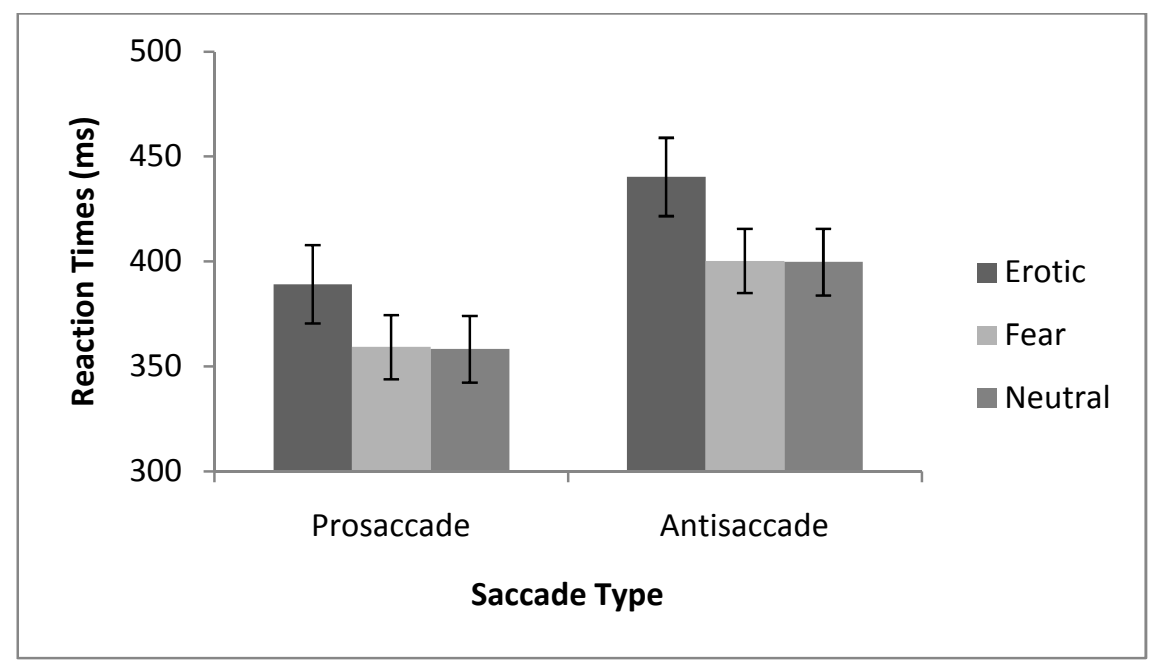

Figure 11: Reaction times for erotic, fearful and neutral pictures in experiment 4. Antisaccades were made significantly slower than prosaccades. Erotic pictures had slower reaction times than the fearful and neutral groups; there was no interaction between picture group and saccade type.

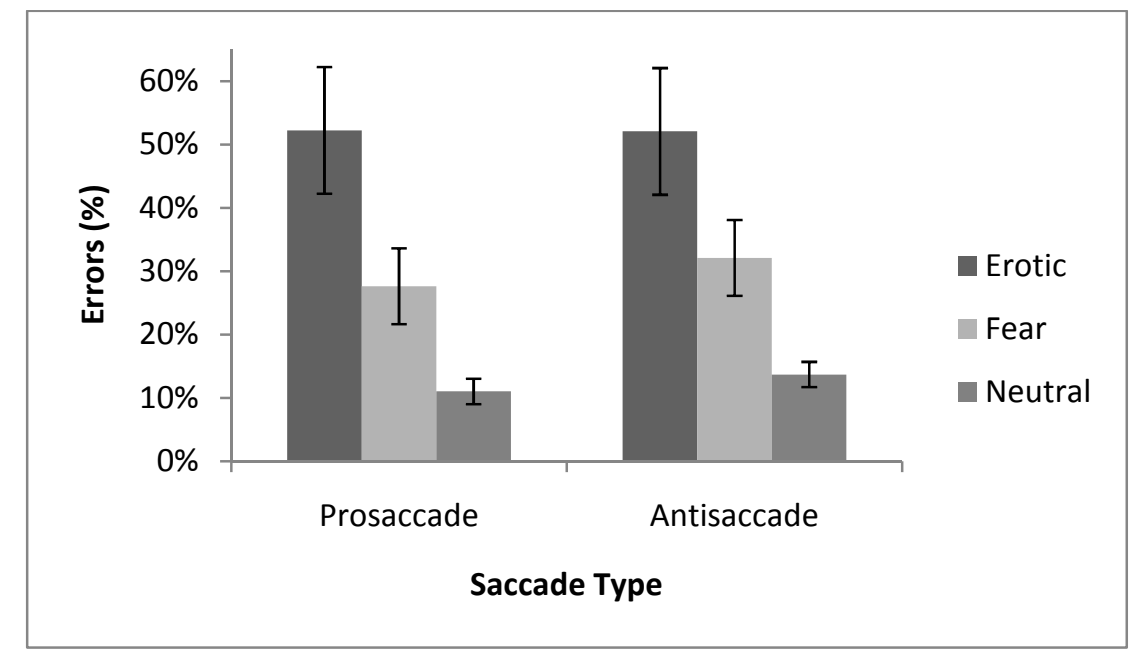

Figure 12: Error rates for erotic, fearful and neutral pictures in experiment 4. There was no difference between the saccade conditions. There were significantly more errors made for the erotic pictures than the fearful pictures; which had more errors than the neutral group. 


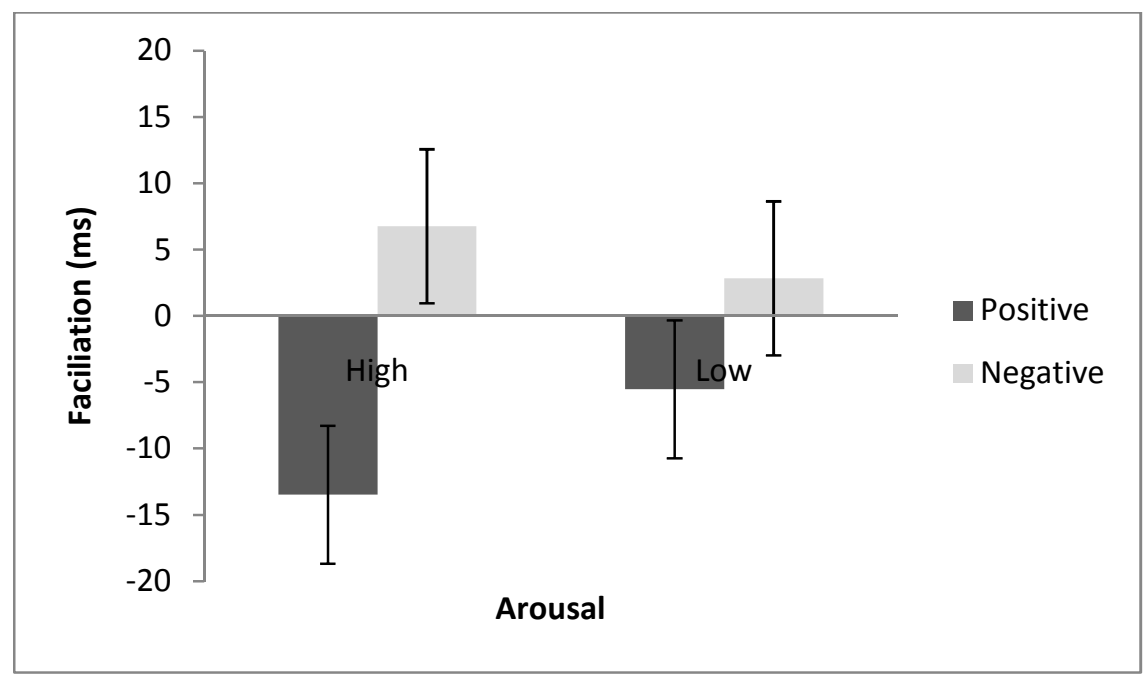

Figure 13: Facilitation provided by each emotional image group for the prosaccade condition for experiment three. Negative valence images facilitated saccade initiation while positive images interfered with initiation.

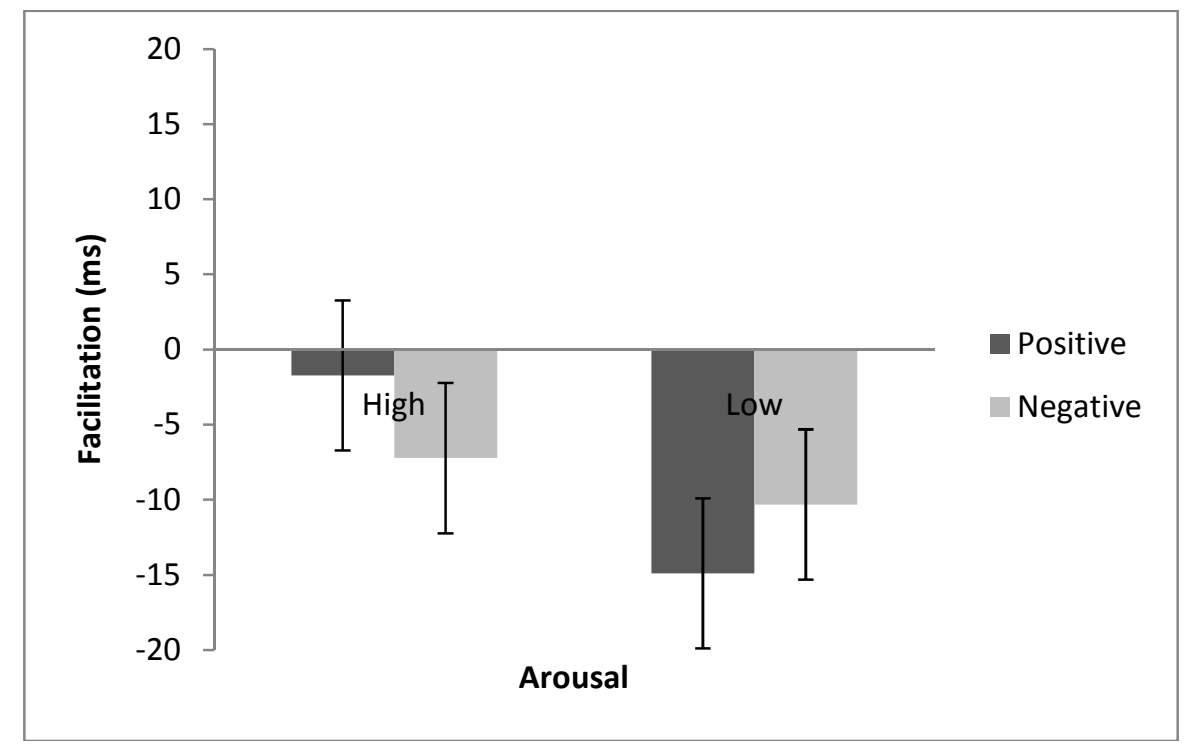

Figure 14: Facilitation provided by each emotional image group for the antisaccade condition for experiment three. The negative images appear to cause more interference than the positive images; however this effect was not significant 


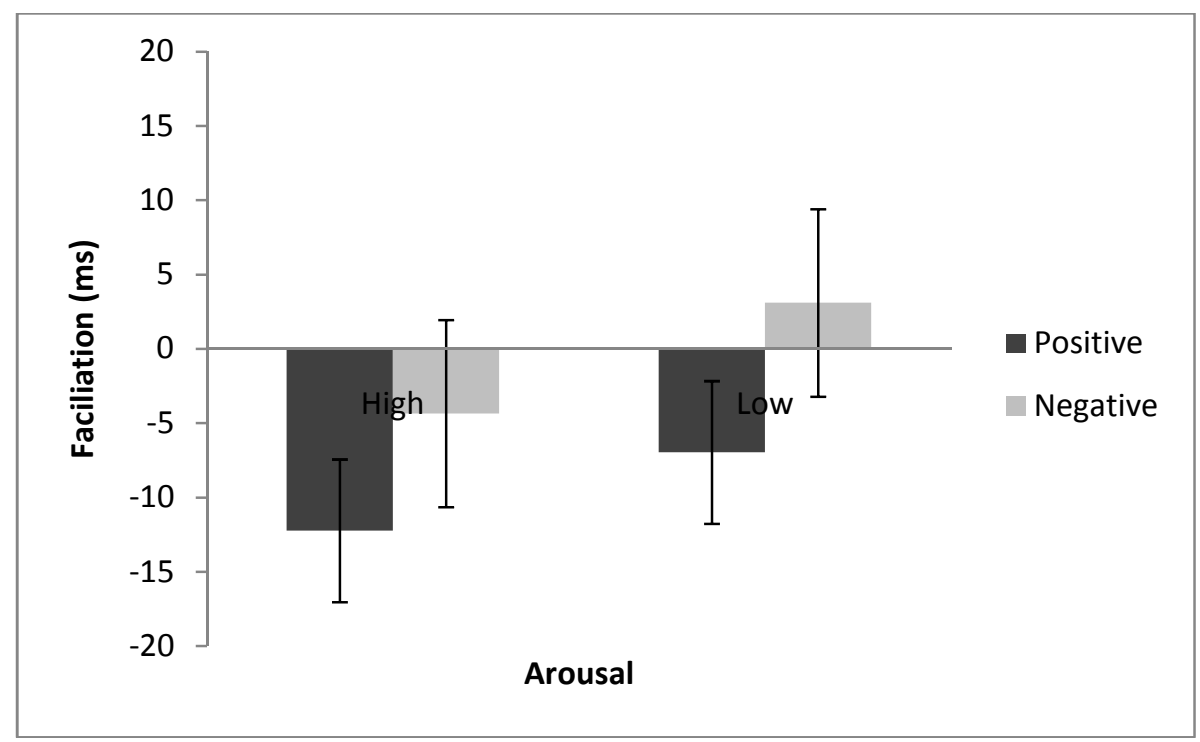

Figure 15: Facilitation provided by each emotional image group for the prosaccade condition for experiment four. Positive valence images interfered with saccade initiation significantly more than the negative valence images

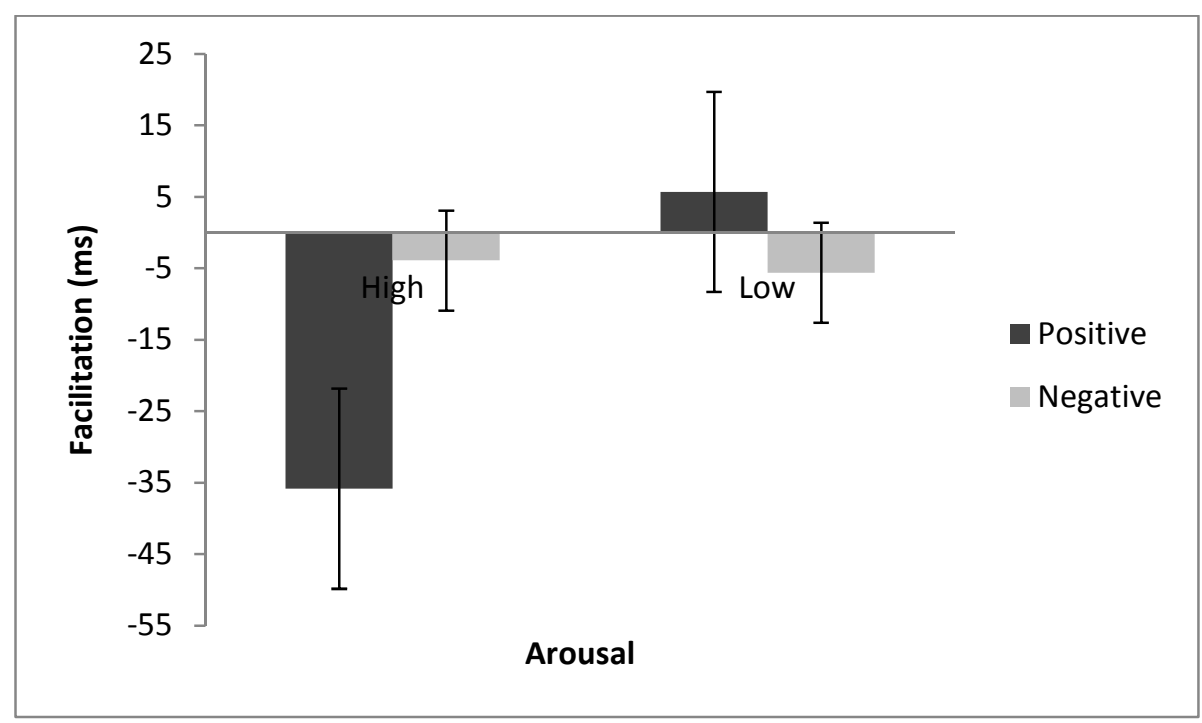

Figure 16: Facilitation provided by each emotional image group for the antisaccade condition for experiment four. There was a significant effect of arousal and an interaction of arousal and valence, driven by the positive valence, high arousal images. 